\title{
ERROR ESTIMATES IN THE FAST MULTIPOLE METHOD FOR SCATTERING PROBLEMS PART 1: TRUNCATION OF THE JACOBI-ANGER SERIES *
}

\author{
Quentin Carayol ${ }^{1}$ and Francis Collino ${ }^{2}$
}

\begin{abstract}
We perform a complete study of the truncation error of the Jacobi-Anger series. This series expands every plane wave $\mathrm{e}^{i \hat{s} \cdot \vec{v}}$ in terms of spherical harmonics $\left\{Y_{\ell, m}(\hat{s})\right\}_{|m| \leq \ell \leq \infty}$. We consider the truncated series where the summation is performed over the $(\ell, m)$ 's satisfying $|m| \leq \ell \leq L$. We prove that if $v=|\vec{v}|$ is large enough, the truncated series gives rise to an error lower than $\epsilon$ as soon as $L$ satisfies $L+\frac{1}{2} \simeq v+C W^{\frac{2}{3}}\left(K \epsilon^{-\delta} v^{\gamma}\right) v^{\frac{1}{3}}$ where $W$ is the Lambert function and $C, K, \delta, \gamma$ are pure positive constants. Numerical experiments show that this asymptotic is optimal. Those results are useful to provide sharp estimates for the error in the fast multipole method for scattering computation.
\end{abstract}

Mathematics Subject Classification. 33C10, 33C55, 41A80.

Received: July 1, 2003.

\section{INTRODUCTION}

\subsection{Motivation}

This paper is the first one of a series of three, addressing the analysis of the error in the fast multipole method (FMM) for scattering problems. Since the pioneer work of Rokhlin, the FMM has been proved to be a very effective tool for solving 3-D acoustic or electromagnetic scattering problems. This method rests on an approximation of the fundamental solution to the Helmholtz equation with a series of multipoles

$$
\frac{\mathrm{e}^{i|u-v|}}{4 \pi i|u-v|} \simeq \sum_{\ell=0}^{L}(2 \ell+1) j_{\ell}(|v|) h_{\ell}^{(1)}(|u|) P_{\ell}\left(\frac{v}{|v|} \cdot \frac{u}{|u|}\right), \quad|v|<|u|,
$$

then on a conversion of the truncated series into a finite sum of plane waves

$$
\frac{\mathrm{e}^{i|u-v|}}{4 \pi i|u-v|} \simeq \frac{1}{4 \pi} f_{S^{2}}\left(\sum_{\ell=0}^{L}(2 \ell+1) i^{\ell} h_{\ell}^{(1)}(|u|) P_{\ell}\left(\frac{v}{|v|} \cdot \frac{u}{|u|}\right)\right) \mathrm{e}^{i v \cdot \hat{s}} \mathrm{~d} \sigma(\hat{s}), \quad|v|<|u| .
$$

Keywords and phrases. Jacobi-Anger, fast multipole method, truncation error.

* Supported by Dassault Aviation.

1 Dassault Aviation, 78, quai Marcel Dassault, Cedex 300, 92552 Saint-Cloud Cedex, France.

e-mail: quentin.carayol@dassault-aviation.fr

2 CERFACS, 42 avenue G. Coriolis, 31057 Toulouse, France. e-mail: Collino@cerfacs.fr 
Here, $j_{\ell}$ and $h_{\ell}^{(1)}$ are the spherical Bessel functions, $P_{\ell}$ the Legendre polynomials, and $f_{S^{2}} \mathrm{~d} \sigma(\hat{s})$ stands for some quadrature rule of the sphere $S^{2}$, see Darve [10] or Chew et al. [5] for more details, and [11] for another multipole formula. The error in this approximation is controlled by both the number of multipoles, $L$, and the choice of the quadrature rule. Greengard and Rokhlin were the first authors to provide empirical laws for the truncation integer $L$ that achieves a given precision, at least when $v$ is not too large. Those formulas have been fixed and improved by Chew and Song [14], (see also Chew [5]), but with no precise analytical error estimates. On the other hand, Rahola [19] then Darve [9], gave precise results, i.e. bounds of the truncation error as function of $L$ but their results lead to overestimate the value of $L$. It is precisely the goal of our study to provide true estimate errors that give the optimal values of $L$, i.e. the ones to be used practically in the FMM. The calculations that we were led to do are rather long and technical [3]. This is the reason why we chose to divide this study into three different parts. In the first one, which constitutes the present article, we study the truncation error in formula (1) when $|u|$ is large, say

$$
\frac{\mathrm{e}^{i|u|}}{4 \pi i|u|} \mathrm{e}^{-i v \cdot \hat{u}} \simeq \frac{\mathrm{e}^{i|u|}}{4 \pi i|u|} \sum_{\ell=0}^{L}(2 \ell+1) j_{\ell}(|v|)(-i)^{\ell} P_{\ell}\left(\frac{v}{|v|} \cdot \hat{u}\right), \quad \hat{u}=\frac{u}{|u|}
$$

It amounts to analyze the Jacobi-anger series. Actually this case has little practical interest, since in most FMM applications $|u|$ and $|v|$ are of same order. Nonetheless we insist on the fact that the analysis of the Jacobi-Anger series is a crucial milestone for the general analysis of the Gegenbauer series written in (1) for finite $|u|$. This importance will particularly appear in a second article to come, which will be devoted to the truncation error for finite $|u|$. Eventually, the error due to the quadrature law in (2) will be the topic of a third article, in which the present results on the Jacobi-Anger series will prove to be very useful again.

After this general presentation, we turn now to the topic of the present paper: the analysis of the truncation of the Jacobi-Anger series. In addition to the motivation presented above, this study presents also some interest by its own: it describes the spectral content of a plane wave in terms of spherical harmonics, and consequently allows us to analyse precisely the far field produced by some potential; this may be useful for Radar Cross Section computation, by providing an efficient way to sample the information. Besides, it can be used to define appropriate parameters in the ultra-weak formulation developed by Cessenat et al. [4].

\subsection{The Jacobi-Anger series}

Let $\hat{s}$ be a unit vector of the unit sphere $S^{2}$ and $\vec{v}$ some vector in $\mathbf{R}^{3}$ with modulus $v$ and direction $\hat{v}: \vec{v}=v \hat{v}$. Jacobi-Anger expansion is, [7],

$$
\mathrm{e}^{i \overrightarrow{\mathbf{v}} \cdot \hat{\mathbf{s}}}=\sum_{\ell=0}^{\infty}(2 \ell+1) i^{\ell} j_{\ell}(v) P_{\ell}(\hat{\mathbf{v}} \cdot \hat{\mathbf{s}})
$$

or

$$
\mathrm{e}^{i \overrightarrow{\mathrm{v}} \cdot \hat{\mathbf{s}}}=4 \pi \sum_{\ell=0}^{\infty} \sum_{m=-\ell}^{\ell} i^{\ell} j_{\ell}(v) Y_{\ell}^{m}(\hat{\mathbf{v}}) \overline{Y_{\ell}^{m}}(\hat{\mathbf{s}}) .
$$

In the expressions above, several special functions take place

- $j_{\ell}(v)$, the spherical Bessel function of order $\ell$. It is linked to the Bessel function via

$$
j_{\ell}(t)=\sqrt{\frac{\pi}{2 t}} J_{\ell+\frac{1}{2}}(t)
$$

- $P_{\ell}(x)$, the Legendre polynomial of order $\ell$. Two important properties of these polynomials are $[15,16]$

$$
P_{\ell}(x) \leq 1, \quad \text { for all } x \in[-1,1], \quad P_{\ell}(1)=1, \quad P_{\ell}(-1)=(-1)^{\ell}
$$



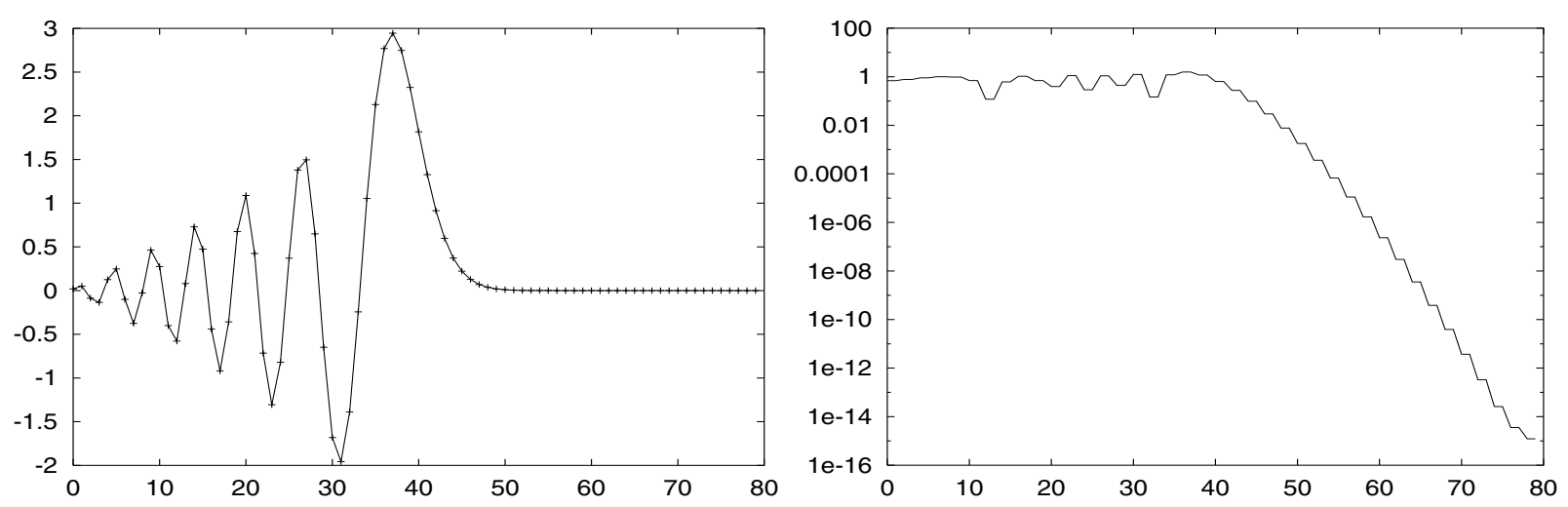

FiguRE 1. Graph of the sequence $(2 \ell+1) j_{\ell}(40)$ versus $\ell$ (left) and truncation error for $v=40$ and $\hat{v} \cdot \hat{s}=1$ versus $L$ (right).

$$
\left.P_{\ell}(x) \leq \sqrt{\frac{2}{\pi\left(\ell+\frac{1}{2}\right)}} \frac{1}{\left(1-x^{2}\right)^{\frac{1}{4}}}, \text { for all } x \in\right]-1,1[.
$$

- $Y_{\ell}^{m}(\hat{s})$, the spherical harmonics of non negative index $\ell$ and of momentum $m, m$ varying from $-\ell$ to $\ell$; the set $\left\{Y_{\ell, m}(\hat{s})\right\}_{|m| \leq \ell \leq \infty}$ forms a complete orthonormal system in $L^{2}\left(S^{2}\right)$. Following the notation of [7], the expression of these functions are given in spherical coordinates by:

$$
Y_{\ell}^{m}(\theta, \varphi)=\sqrt{\frac{2 \ell+1}{4 \pi} \frac{(\ell-|m|) !}{(\ell+|m|) !}} P_{\ell}^{|m|}(\cos \theta) \mathrm{e}^{i m \varphi},
$$

where $P_{\ell}^{m}(t)$ are the associated Legendre functions defined by

$$
P_{\ell}^{m}(x)=\left(1-x^{2}\right)^{\frac{m}{2}} \frac{\mathrm{d}^{m} P_{\ell}(x)}{\mathrm{d} x^{m}}, m \geq 0
$$

The equivalence between (3) and (4) comes from the addition formula

$$
(2 \ell+1) P_{\ell}^{m}(\hat{\mathbf{v}} \cdot \hat{\mathbf{s}})=4 \pi \sum_{m=-\ell}^{\ell} Y_{\ell}^{m}(\hat{\mathbf{v}}) \overline{Y_{\ell}^{m}}(\hat{\mathbf{s}}) .
$$

For a precise definition of these functions and of their properties, the reader can refer to $[1,7,12,17,21]$ for instance.

Let $L$ be some positive integer. We will use $L$ to truncate the series. The truncated series is the $L^{2}\left(S^{2}\right)$ projection onto the linear space spanned by the spherical harmonics of degree less or equal to $L$. Our goal is to study the error of truncation with respect to $L$.

A first property is that $L$ must always be chosen greater than $v$, at least if a small enough error $\epsilon$ is required. To illustrate that, we give a particular example, representative of general cases. We choose $v=40$ and $\hat{s}$ directed in the same direction as $\vec{v}$. In Figure 1 , we show the variations of the sequence $(2 \ell+1) j_{\ell}(40)$ versus $\ell$ as well as the truncation error with respect to $L$. We observe that the sequence $(2 \ell+1) j_{\ell}(40)$ begins with mild oscillations that stop at some integer $\ell_{40} \approx 45>v$, besides, the sequence approaches 0 very rapidly. At the same time, the error decreases in a significant manner only when $L$ overpasses the integer $\ell_{40}$, i.e. when $(2 \ell+1) j_{\ell}(40)$ is no longer oscillating. From $L=\ell_{40}$, the decrease seems to be rather fast, apparently more than exponential. The analysis of these curves shows that it is especially interesting to study the error for $L$ slightly larger than $v$. 
We will always assume that

$$
L+\frac{1}{2} \geq v
$$

In many papers, empirical formulas can be found in the form $L=v+C(\epsilon) \ln (\pi+v)$, or, sometimes, $L=$ $v+C(\epsilon) v^{\frac{1}{3}}$, to obtain a truncation error lower than $\epsilon$, (see [5,6] for instance). Let us show some numerical experiments applying these formulas. We compute numerically the error

$$
e_{j a}(L, \vec{v}, \hat{s})=\left|\sum_{\ell=L+1}^{\infty}(2 \ell+1) i^{\ell} j_{\ell}(v) P_{\ell}(\hat{\mathbf{v}} \cdot \hat{\mathbf{s}})\right|
$$

for 3 different angular apertures, $\hat{\mathbf{v}} \cdot \hat{\mathbf{s}}=1, \hat{\mathbf{v}} \cdot \hat{\mathbf{s}}=0$ and $\hat{\mathbf{v}} \cdot \hat{\mathbf{s}}=0.5$. The results are presented in Figure 2 .
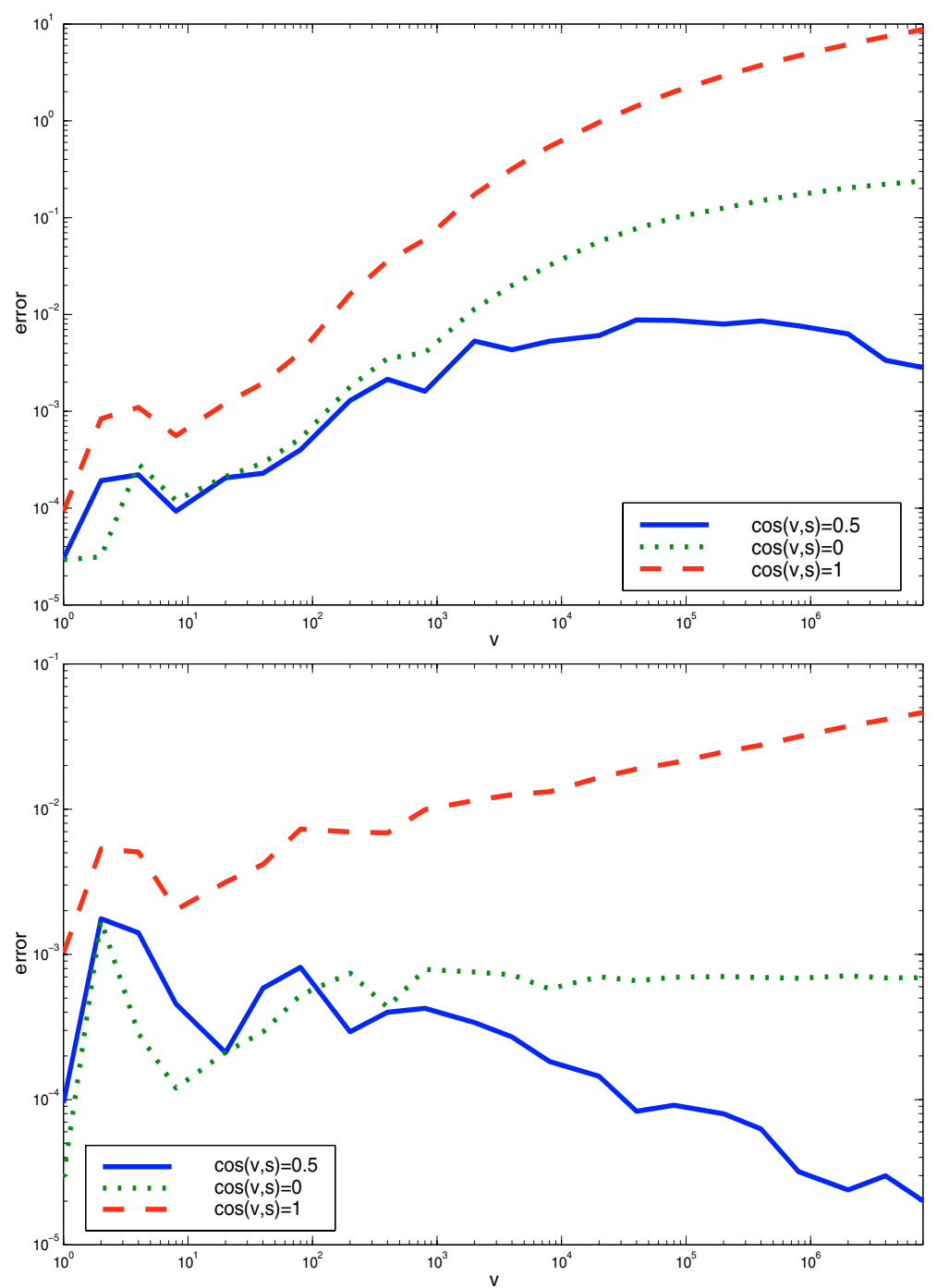

Figure 2. Truncation error for the formula $L=v+3 \ln (v+\pi)$ (top) or for the formula $L=v+3 v^{\frac{1}{3}}$ (bottom) versus $v, \hat{\mathbf{v}} \cdot \hat{\mathbf{s}}$ is kept constant, equal to $0,0.5$ and 1 . 
Figure 2 shows that formula $L=v+C(\epsilon) \ln (\pi+v)$ does not provide a constant error in $v$; for large $v$, the truncation error increases when $\hat{\mathbf{v}} \cdot \hat{\mathbf{s}}=1$ or 0 but decreases when $\hat{\mathbf{v}} \cdot \hat{\mathbf{s}}=0.5$. The law $L=v+C(\epsilon) v^{\frac{1}{3}}$ seems to be better; the error seems to be asymptotically constant for $\hat{\mathbf{v}} \cdot \hat{\mathbf{s}}=0$ and decreases when $\hat{\mathbf{v}} \cdot \hat{\mathbf{s}}=0.5$. However, the error increases mildly, apparently in $v^{\frac{1}{6}}$ when $\hat{\mathbf{v}} \cdot \hat{\mathbf{s}}=1$. All these properties will be recovered theoretically in Section 3.1.

The aim of this work is to perform a systematic analysis of the truncation error and especially to provide an asymptotic formula as $v$ goes to infinity. We will also analyze the series

$$
e_{j a}^{a b s}(L, v)=\sum_{\ell=L+1}^{\infty}(2 \ell+1)\left|j_{\ell}(v)\right|
$$

which constitutes, according to (6), a uniform upper bound of $e_{j a}(L, \vec{v}, \hat{s})$. The results that we will obtain for this case will be used intensively in the forthcoming paper when we will focus on the analysis of the truncation error in the addition formula (1).

The main results of the paper can be described in the following way. We derive laws in the form $L_{a b s}(v, \epsilon)$ and $L_{\beta}(v, \epsilon)$ such that

$$
\sum_{\ell=L_{\mathrm{abs}}(v, \epsilon)+1}^{\infty}(2 \ell+1)\left|j_{\ell}(v)\right| \leq \epsilon, \quad\left|\sum_{\ell=L_{\beta}(v, \epsilon)+1}^{\infty}(2 \ell+1) i^{\ell} j_{\ell}(v) P_{\ell}(\cos \beta)\right| \leq \epsilon
$$

with the asymptotics (subscript $*$ stands for abs or $\beta$ )

$$
L_{*}(v, \epsilon) \simeq v+C_{*} W^{\frac{2}{3}}\left(K_{*} \frac{v^{\gamma_{*}}}{\epsilon^{\delta_{*}}}\right) v^{\frac{1}{3}}-\frac{1}{2} \text { plus terms vanishing with } v \text { when } v \text { is large, }
$$

with constants specific to the considered case. Here, function $W$ is the Lambert function $W(t) \mathrm{e}^{W(t)}=t$ satisfying $W(t) \simeq \log (t / \log t)$ when $t$ is large, see Figure 5. More precisely, we will get:

\begin{tabular}{|c||c|c|c|c|c|}
\hline & $C_{*}$ & $K_{*}$ & $\gamma_{*}$ & $\delta_{*}$ & $c f$. formula \\
\hline \hline$L_{a b s}(v, \epsilon):$ & $\frac{1}{2}\left(\frac{3}{2}\right)^{\frac{2}{3}}$ & $\frac{2}{3}$ & 1 & 2 & $(32)$ \\
\hline$L_{\beta}(v, \epsilon), \cos \beta= \pm 1:$ & $\left(\frac{1}{2}\right)^{\frac{5}{3}}$ & $\frac{1}{4}$ & 1 & 6 & $(52)$ \\
\hline$L_{\beta}(v, \epsilon), \cos \beta=0$ & $\frac{1}{2}\left(\frac{3}{2}\right)^{\frac{2}{3}}$ & $\frac{1}{3 \pi}$ & 0 & 2 & $(40)$ \\
\hline$L_{\beta}(v, \epsilon), \cos \beta \neq 0, \pm 1$ & $\frac{1}{2}\left(\frac{3}{2}\right)^{\frac{2}{3}}$ & $\frac{2}{3 \pi|\sin \beta|}$ & 0 & 2 & $(42)$ \\
\hline
\end{tabular}

These laws appear to be asymptotically optimal in the three first cases. The last case $(\cos \beta \neq-1,0$ or 1$)$ that corresponds to the last line of the array above is not optimal since taking $L$ larger than $v$ gives an error less than $\epsilon$ for large enough $v$ (cf. the result of Prop. 3.1). 
Furthermore, we prove that the law corresponding to $\beta=0$ (or $\pi$ ), i.e.

$$
L_{0}(v, \epsilon)=\frac{v}{x_{\epsilon}(v)}-\frac{1}{2}, \text { with } x_{\epsilon}(v) \text { solution of } \frac{\left(1-x_{\epsilon}(v)^{2}\right)^{\frac{3}{2}}}{x_{\epsilon}(v)}=\frac{1}{2 v} W\left(\frac{v}{4 \epsilon^{6}}\right),
$$

(and whose asymptotic reads in the second line of the array) gives the bound

$$
\sup _{\beta}\left|\sum_{\ell=L_{0}(v, \epsilon)+1}^{\infty}(2 \ell+1) i^{\ell} j_{\ell}(v) P_{\ell}(\cos \beta)\right| \leq \epsilon+\varepsilon\left(\frac{v}{4 \epsilon^{6}}\right) \text {, with } \varepsilon(\xi)=2 C_{0} \xi^{-1 / 6} W^{-1 / 3}(\xi),
$$

where $C_{0}$ is the pure constant defined in (53). Note that the term $\varepsilon\left(v / 4 \epsilon^{6}\right)$ goes to zero when $v$ goes to infinity or when $\epsilon$ approaches zero. As an illustration of this result, we have the following corollary

Proposition 1.1. Let $q$ be in $L^{1}\left(\mathbf{R}^{3}\right)$ compactly supported in $B_{R}$ the ball of center 0 and radius $R$, let $\hat{x}$ be some unit vector, and $k$ some real wavenumber, then the far field

$$
a_{\infty}(\hat{x})=\frac{1}{4 \pi} \int_{B_{R}} q(y) \mathrm{e}^{-i k \hat{x} \cdot y} \mathrm{~d} y
$$

associated to the potential

$$
u(x)=\frac{1}{4 \pi} \int_{B_{R}} \frac{\mathrm{e}^{i k|x-y|}}{|x-y|} q(y) \mathrm{d} y
$$

can be approximated with the spherical function

$$
a_{\infty}^{L}(\hat{x})=\sum_{\ell=0}^{L} \sum_{m=-\ell}^{\ell}\left(\int_{B_{R}} i^{\ell} j_{\ell}(k|y|) \bar{Y}_{\ell}^{m}(\widehat{y}) \mathrm{d} y\right) Y_{\ell}^{m}(\hat{x})
$$

with the bound

$$
\sup _{\hat{x}}\left|a_{\infty}^{L}(\hat{x})-a_{\infty}(\hat{x})\right| \leq\left(\epsilon+\varepsilon\left(\frac{k R}{4 \epsilon^{6}}\right)\right) \frac{\|q\|_{L^{1}}}{4 \pi}
$$

as soon as $L$ satisfies to

$$
\begin{aligned}
& L>L_{0}(k R, \epsilon), L_{0}(v, \epsilon) \text { given in (12): } \\
& L_{0}(k R, \epsilon)=k R+\left(\frac{1}{2}\right)^{5 / 3} W^{2 / 3}\left(\frac{k R}{4 \epsilon^{6}}\right)(k R)^{1 / 3}-\frac{1}{2}+\ldots
\end{aligned}
$$

Since linear combinations of spherical harmonic functions of degree less or equal to $L$ can be exactly recovered through the knowledge of $2 L^{2}$ points (see [10]), this result provides us with a precise rule to sample farfields according to the size of the perturbation.

Our paper is organized as follows. First in Section 2 the series in (11) is analyzed. For a given precision $\epsilon$, one gives a $L^{\epsilon}(v)$ for which the truncation error is lower than $\epsilon$ for $L \geq L^{\epsilon}(v)$; then, we carry on by performing the analysis of the asymptotic behavior of $L^{\epsilon}(v)$ for large $v$. In Section 3, the truncation error (10) is studied. Three cases are discussed according to whether $\vec{v}$ and $\vec{s}$ point in the same direction, are perpendicular or present other configurations. Once again, a truncation law $L^{\epsilon}(\vec{v}, \hat{s})$ is derived and asymptotic laws are given. A uniform bound, improving the results derived in the first section, is also given. Finally, Section 4 gives some estimates on the $L^{2}\left(S^{2}\right)$ error. 


\section{Analysis of the truncation error for the SERIES (11)}

In this section, we analyse the series $e_{j a}^{a b s}(L, v)$ assuming that $v \leq L+\frac{1}{2}$. This restriction allows us to get rid of the absolute values since

$$
j_{\ell}(v)=\sqrt{\frac{\pi}{2 v}} J_{\ell+\frac{1}{2}}(v)>0, \text { if } v \leq L+\frac{1}{2}<j_{L+\frac{1}{2}, 0}
$$

where $j_{\nu, 0}$ denotes the first zero of the function $J_{\nu}(v)$ (the property $j_{\nu, 0} \geq \nu+1$, when $\nu$ is larger than $\frac{1}{2}$ can be found for instance in [21]). We have consequently

$$
\left\{\begin{array}{c}
e_{j a}^{a b s}(L, v)=\epsilon_{1}(L, v)+\epsilon_{2}(L, v) \\
\epsilon_{1}(L, v)=\sum_{\substack{l=L+1+p \\
p \geq 0 \text { even }}}(2 \ell+1) j_{\ell}(v), \quad \epsilon_{2}(L, v)=\sum_{\substack{\ell=L+1+p \\
p \geq 0 \text { odd }}}(2 \ell+1) j_{\ell}(v) .
\end{array}\right.
$$

We distinguish even integers from odd integers in the summation only for technical reasons; this splitting corresponds to studying separately the truncation error on the real and imaginary parts of the Jacobi-Anger series, $c f .(3)$.

\subsection{Estimates}

Lemma 2.1. Let $v$ be a positive real number and $L$ be a non negative integer. We have

$$
\left\{\begin{array}{l}
\epsilon_{1}(L, v)=\sqrt{\frac{\pi v}{2}}\left(L+\frac{1}{2}\right) \int_{0}^{v} J_{L+\frac{1}{2}}(u) \frac{\mathrm{d} u}{u} \\
\epsilon_{2}(L, v)=\sqrt{\frac{\pi v}{2}}\left(L+\frac{3}{2}\right) \int_{0}^{v} J_{L+\frac{3}{2}}(u) \frac{\mathrm{d} u}{u}
\end{array}\right.
$$

Moreover, if $v \leq L+\frac{1}{2}$,

$$
\epsilon_{2}(L, v) \leq \frac{v}{L+\frac{1}{2}} \epsilon_{1}(L, v) \leq \epsilon_{1}(L, v) .
$$

Proof. From Definitions (13) and (5), we have

$$
\epsilon_{1}(L, v)=\sqrt{\frac{\pi}{2 v}} \sum_{\substack{l=L+1+p \\ p \text { even }}} 2\left(l+\frac{1}{2}\right) J_{l+\frac{1}{2}}(v),
$$

or, using the equality

$$
\begin{gathered}
2 \nu J_{\nu}(v)=v\left(J_{\nu+1}(v)+J_{\nu-1}(v)\right), \\
\epsilon_{1}(L, v)=\sqrt{\frac{\pi v}{2}} \sum_{p=0}^{\infty}\left(J_{L+2 p+\frac{1}{2}}(v)+J_{L+2 p+\frac{5}{2}}(v)\right) .
\end{gathered}
$$

Let $f_{p}(v)$ be $J_{L+2 p+\frac{1}{2}}(v)+J_{L+2 p+\frac{5}{2}}(v)$. Since

$$
2 J_{\nu}^{\prime}(v)=J_{\nu-1}(v)-J_{\nu+1}(v),
$$

the derivative of $f_{p}(v)$ is

$$
f_{p}^{\prime}(v)=\frac{1}{2}\left(J_{L+2 p-\frac{1}{2}}(v)-J_{L+2 p+\frac{7}{2}}(v)\right),
$$


and

$$
\epsilon_{1}(L, v)=\sqrt{\frac{\pi v}{2}} \sum_{p=0}^{\infty} \int_{0}^{v} \frac{1}{2}\left(J_{L+2 p-\frac{1}{2}}(u)-J_{L+2 p+\frac{7}{2}}(u)\right) \mathrm{d} u=\sqrt{\frac{\pi v}{2}} \int_{0}^{v} \frac{1}{2}\left(J_{L-\frac{1}{2}}(u)+J_{L+\frac{3}{2}}(u)\right) \mathrm{d} u .
$$

Using once again (17), we get the first equality of (14). The second one can be obtained in a similar way. Finally, (15) is a direct consequence of the following inequality ([2], p. 26),

$$
J_{L+\frac{3}{2}}(u) \leq \frac{u}{L+\frac{3}{2}} J_{L+\frac{1}{2}}(u), \quad \text { for } u<L+\frac{3}{2} .
$$

This ends the proof.

We infer from inequality (15) that

$$
e_{j a}^{a b s}(L, v) \leq 2 \epsilon_{1}(L, v) .
$$

Therefore, we only need to study the behaviour of $\epsilon_{1}(L, v)$. We introduce the notation

$$
\nu=L+\frac{1}{2}, x=\frac{v}{L+\frac{1}{2}},
$$

and use the change of variables $u=\nu s$ in (14) to get

$$
\epsilon_{1}(L, v)=\sqrt{\frac{\pi v}{2}} \int_{0}^{\frac{v}{\nu}} \nu J_{\nu}(\nu s) \frac{\mathrm{d} s}{s} .
$$

Now, we recall the following estimate due to Watson ([21], p. 255), [20],

$$
\forall \nu>0, \quad \forall s \in] 0,1\left[, \quad J_{\nu}(\nu s) \leq \frac{\mathrm{e}^{-\nu F(s)}}{\left(1-s^{2}\right)^{\frac{1}{4}} \sqrt{2 \pi \nu}}\right.
$$

where the function $F$ is defined by

$$
F(s)=\ln \left(1+\sqrt{1-s^{2}}\right)-\ln (s)-\sqrt{1-s^{2}} .
$$

We use inequality (22) in (21) and obtain

$$
\epsilon_{1}(L, v) \leq \frac{\sqrt{x}}{2} \int_{0}^{x} \frac{\nu \mathrm{e}^{-\nu F(s)}}{s\left(1-s^{2}\right)^{\frac{1}{4}}} \mathrm{~d} s .
$$

Since $F^{\prime}(s)=-\frac{\sqrt{1-s^{2}}}{s}$, we have that

$$
\epsilon_{1}(L, v)=\frac{\sqrt{x}}{2} \int_{0}^{x}\left(-\nu F^{\prime}(s)\right) \frac{\mathrm{e}^{-\nu F(s)}}{\left(1-s^{2}\right)^{\frac{3}{4}}} \mathrm{~d} s, \leq \frac{\sqrt{x}}{2\left(1-x^{2}\right)^{\frac{3}{4}}}\left[\mathrm{e}^{-\nu F(s)}\right]_{0}^{x}=\frac{\sqrt{x}}{2\left(1-x^{2}\right)^{\frac{3}{4}}} \mathrm{e}^{-\nu F(x)} .
$$

Using $v$ and $x$ as independent variables, this inequality reads

$$
\epsilon_{1}(L, v) \leq \frac{\sqrt{x}}{2\left(1-x^{2}\right)^{\frac{3}{4}}} \mathrm{e}^{-v \frac{F(x)}{x}}=G(v, x) .
$$


For $0<x<1$, the derivative of $\frac{F(x)}{x}$ is

$$
\left(\frac{F(x)}{x}\right)^{\prime}=-\frac{1}{x^{2}}\left[\ln \left(1+\sqrt{1-x^{2}}\right)-\ln x\right]<0
$$

which proves that $x \rightarrow G(v, x)$ is a one to one mapping and an increasing function from $] 0,1[$ onto $] 0,+\infty[$. Let $x_{\epsilon}(v)$ be the unique solution of the equation $G\left(v, x_{\epsilon}(v)\right)=\epsilon$ for given $(v, \epsilon)$. We have seen that $\epsilon_{1}(L, v) \leq \epsilon$ for all $L$ greater than or equal to $L^{\epsilon}(v)=\frac{v}{x_{\epsilon}(v)}-\frac{1}{2}$. The function $x \rightarrow G(v, x)$ being non decreasing, it is enough to proceed by dichotomy to get a simple algorithm for the determination of a possible truncation value.

Thus, we have established the following proposition:

Proposition 2.2. Let $v, \epsilon$ be two real positive numbers. Let us define

$$
L_{\mathcal{G}}^{\epsilon}(v)=\frac{v}{x_{\epsilon}(v)}-\frac{1}{2}
$$

where $x_{\epsilon}(v)$ is the unique solution in $] 0,1[$ of the equation

$$
G\left(v, x_{\epsilon}(v)\right)=\epsilon, \quad\left(\mathcal{C}_{\mathcal{G}} \text { criteria }\right)
$$

with

$$
G(v, x)=\frac{\sqrt{x}}{2\left(1-x^{2}\right)^{\frac{3}{4}}} \mathrm{e}^{-v \frac{F(x)}{x}}, \quad F(x)=\ln \left(1+\sqrt{1-x^{2}}\right)-\ln (x)-\sqrt{1-x^{2}}
$$

then, we have

$$
\epsilon_{1}(L, v)=\sum_{\substack{l=L+1+p \\ p \text { even }}}(2 l+1) j_{l}(v) \leq \epsilon \text { and } e_{j a}^{a b s}(L, v)=\sum_{\ell=L+1}^{\infty}(2 \ell+1)\left|j_{\ell}(v)\right| \leq 2 \epsilon
$$

as soon as $L$ is greater than or equal to $L_{\mathcal{G}}^{\epsilon}(v)$.

We verify numerically that $G(v, x(L, v))$ is a good approximation of $\epsilon_{1}(L, v)$. In Figure 3 , the variations of $Q(v, L)$, ratio of $\epsilon_{1}(L, v)$ with $G\left(v, \frac{v}{L+\frac{1}{2}}\right)$, are shown for different values of $v$ when $L$ goes beyond $v$. We see that the precision is improved as $L$ increases, but seems to deteriorate when $v$ is large. This is not very important since we will show that the truncation corresponding to a small error must be done beyond $v+C v^{\frac{1}{3}}$. A more pertinent analysis consists in looking at the variations of $Q(v, L)$ when $L=v+C v^{\frac{1}{3}}$. It is what is shown in Figure 4. We observe a constant precision, improving as $C$ increases. Our estimates should be optimal as $v$ goes to infinity.

\subsection{Estimates for large $\boldsymbol{v}$. Asymptotic behaviour}

For very large $v$, it is clear that the ratio $x=\frac{v}{L+\frac{1}{2}}$ must approach 1 if the estimated error $G\left(v, \frac{v}{L+\frac{1}{2}}\right)$ is $\operatorname{kept}$ constant. Expanding in series the logarithm involved in Definition (23), we get

$$
\forall x \in] 0,1], \quad F(x)=\sum_{p=1}^{+\infty}{\frac{\left(\sqrt{1-x^{2}}\right)^{2 p+1}}{2 p+1}}^{2}
$$

and

$$
F(x) \geq \frac{1}{3}\left(1-x^{2}\right)^{\frac{3}{2}}
$$




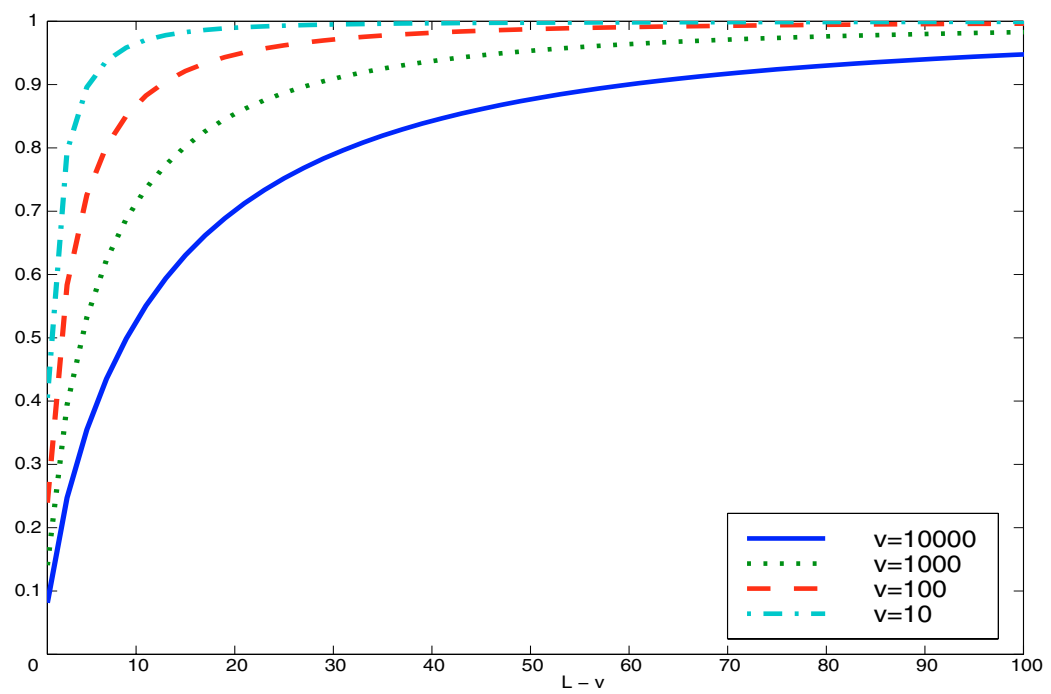

Figure 3. Quality factor for $\epsilon_{1}(L, v)$ when $v$ is given.

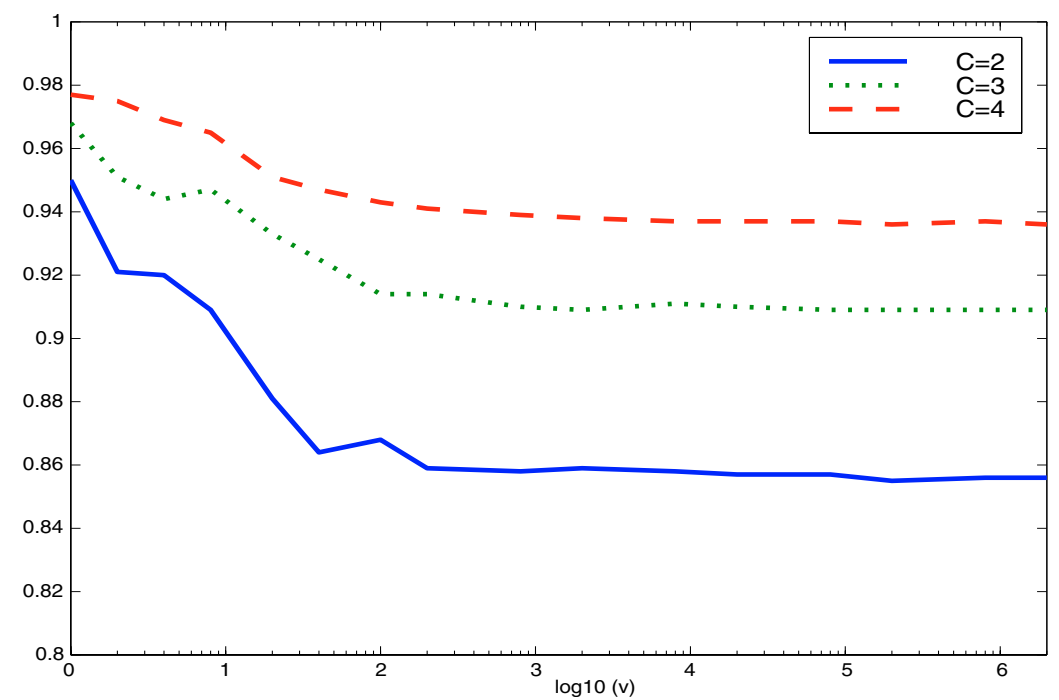

Figure 4. Quality factor for $\epsilon_{1}(L, v)$ when $L=v+C v^{\frac{1}{3}}$.

We substitute to criterion $\left(\mathcal{C}_{\mathcal{G}}\right)$ the following weaker criterion, denoted by $\left(\mathcal{C}_{\mathcal{H}}\right)$

$$
H\left(v, x_{\epsilon}(v)\right)=\epsilon, \quad \text { with } \quad H(v, x)=\frac{\sqrt{x}}{2\left(1-x^{2}\right)^{\frac{3}{4}}} \mathrm{e}^{-v \frac{\left(1-x^{2}\right)^{\frac{3}{2}}}{3 x}}
$$


Let us define $\Theta_{\epsilon}(v)=\frac{\left(1-x_{\epsilon}^{2}(v)\right)^{\frac{3}{2}}}{x_{\epsilon}(v)}$. Equality (24) becomes

$$
\frac{\mathrm{e}^{-v \frac{\Theta_{\epsilon}(v)}{3}}}{2 \sqrt{\Theta_{\epsilon}(v)}}=\epsilon \Longleftrightarrow \frac{2}{3} v \Theta_{\epsilon}(v) \mathrm{e}^{\frac{2}{3} v \Theta_{\epsilon}(v)}=\frac{v}{6 \epsilon^{2}}
$$

At this point, we introduce the Lambert function $W(t)[8]$, defined on the interval $\left[-\frac{1}{e},+\infty[\right.$ by

$$
W(t) e^{W(t)}=t
$$

and whose graph is shown in Figure 5 . We have the asymptotic behavior for $t \rightarrow+\infty$

$$
W(t)=\log \left(\frac{t}{\log t}\right)+o(1)
$$

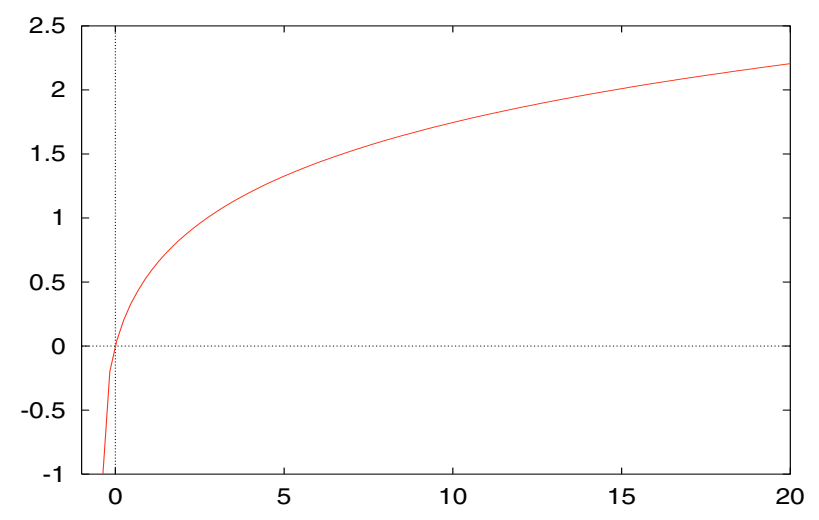

Figure 5. Graph of the Lambert function $W, W(t) e^{W(t)}=t$.

Using this function, Criterion $\left(\mathcal{C}_{\mathcal{H}}\right)$, i.e. $(25)$, can be rewritten in the form

$$
\Theta_{\epsilon}(v)=\frac{3}{2 v} W\left(\frac{v}{6 \epsilon^{2}}\right) .
$$

We have

$$
\lim _{v \rightarrow+\infty} \Theta_{\epsilon}(v)=0 \text { and } \lim _{v \rightarrow+\infty} x_{\epsilon}(v)=1^{-},
$$

and a rather simple calculation permits us to obtain the asymptotic expansion of $x_{\epsilon}(v)$ :

$$
x_{\epsilon}(v)=1-\frac{1}{2}\left(\frac{3}{2 v}\right)^{\frac{2}{3}} W^{\frac{2}{3}}\left(\frac{v}{6 \epsilon^{2}}\right)+\mathcal{O}\left(v^{-\frac{4}{3}} W^{\frac{4}{3}}\left(\frac{v}{6 \epsilon^{2}}\right)\right)
$$

then, to obtain the asymptotic expansion of $\nu_{\epsilon}(v)$

$$
\nu_{\epsilon}(v)=L_{\epsilon}(v)+\frac{1}{2}=v+\frac{1}{2}\left(\frac{3}{2}\right)^{\frac{2}{3}} v^{\frac{1}{3}} W^{\frac{2}{3}}\left(\frac{v}{6 \epsilon^{2}}\right)+\mathcal{O}\left(v^{-\frac{1}{3}} W^{\frac{4}{3}}\left(\frac{v}{6 \epsilon^{2}}\right)\right) .
$$


Thus, we have established the following proposition:

Proposition 2.3. Let $v, \epsilon$ be two positive real numbers. For every positive integer $L \geq v-\frac{1}{2}$, we have

$$
\sum_{\ell=L+1}^{\infty}(2 \ell+1)\left|j_{\ell}(v)\right| \leq \frac{\sqrt{x}}{\left(1-x^{2}\right)^{\frac{3}{4}}} \mathrm{e}^{-v \frac{\left(1-x^{2}\right)^{\frac{3}{2}}}{3 x}}, \quad x=\frac{v}{L+\frac{1}{2}} .
$$

Moreover, if we define

$$
L_{\mathcal{H}}^{\epsilon}(v)=\frac{v}{x_{\epsilon}(v)}-\frac{1}{2}
$$

where $x_{\epsilon}(v)$ is the unique solution in $] 0,1[$ of the equation

$$
\frac{\left(1-x_{\epsilon}(v)^{2}\right)^{\frac{3}{2}}}{x_{\epsilon}(v)}=\frac{3}{2 v} W\left(\frac{2 v}{3 \epsilon^{2}}\right) \quad\left(\text { criteria }\left(\mathcal{C}_{\mathcal{H}}\right)\right),
$$

then, the estimates

$$
\left\{\begin{array}{l}
\epsilon_{1}(L, v)=\sum_{\substack{l=L+1+p \\
p \text { even } \\
\text { even }}}(2 l+1) j_{l}(v) \leq \frac{\epsilon}{2}, \\
e_{j a}^{a b s}(L, v)=\sum_{\ell=L+1}^{\infty}(2 \ell+1)\left|j_{\ell}(v)\right| \leq \epsilon,
\end{array}\right.
$$

hold as soon as $L$ is greater than $L_{\mathcal{H}}^{\epsilon}(v)$. Furthermore, if we set

$$
L^{\epsilon, \infty}(v)+\frac{1}{2}=v+\frac{1}{2}\left(\frac{3}{2}\right)^{\frac{2}{3}} W^{\frac{2}{3}}\left(\frac{2 v}{3 \epsilon^{2}}\right) v^{\frac{1}{3}}
$$

then

and

$$
L_{\mathcal{H}}^{\epsilon}(v)=L^{\epsilon, \infty}(v)\left(1+\mathcal{O}\left(v^{-\frac{4}{3}} W^{\frac{4}{3}}\left(\frac{v}{6 \epsilon^{2}}\right)\right)\right)
$$

$$
\lim _{v \rightarrow \infty} \sum_{\ell=L^{\epsilon, \infty}(v)+1}^{\infty}(2 \ell+1)\left|j_{\ell}(v)\right| \leq \lim _{v \rightarrow \infty} 2 \epsilon_{1}\left(L^{\epsilon, \infty}(v), v\right) \leq \epsilon .
$$

Remark 2.4. It is possible to solve (31) explicitly. We have

$$
\left.\left(1-x_{\epsilon}^{2}\right)^{3}=\Theta^{2} x_{\epsilon}^{2}, x_{\epsilon} \in\right] 0,1\left[\Leftrightarrow \quad x_{\epsilon}=\sqrt{1+\frac{\delta}{6}-2 \frac{\Theta^{2}}{\delta}}, \text { with } \delta=\left(12 \sqrt{12 \Theta^{6}+81 \Theta^{4}}-108 \Theta^{2}\right)^{\frac{1}{3}} .\right.
$$

Remark 2.5. A priori, formula (32) is only an asymptotic estimate. It is nevertheless efficient even for rather small values of $v$. In Figure 6 is shown the difference $\Delta L$ between $L(v, \epsilon)$ corresponding to the smallest $L$ for which $\epsilon_{1}(L, v)<\epsilon$ holds, and $L^{\frac{\epsilon}{2}, \infty}(v)$. The two cases $\epsilon=10^{-3}$ and $\epsilon=10^{-7}$ are considered. For $v>10,|\Delta L|$ does not overpass 1 !

\section{Analysis of the trunchtion for the Jacobi-Anger Series}

The calculations of the previous section provide uniform estimates of the remainder of the truncated JacobiAnger series, uniformly with respect to the directions $\hat{s}$ and $\hat{v}$. In this section, we show that we can improve the estimates by taking into account the Legendre polynomials. In a first subsection, we begin with the analysis of the truncation error when the aperture angle $\beta, \cos \beta=\hat{s} \cdot \hat{v}$, is kept constant. We distinguish 3 cases according to whether $|\cos \beta|$ is equal to 0,1 or is different from these two values. We are essentially interested in the 

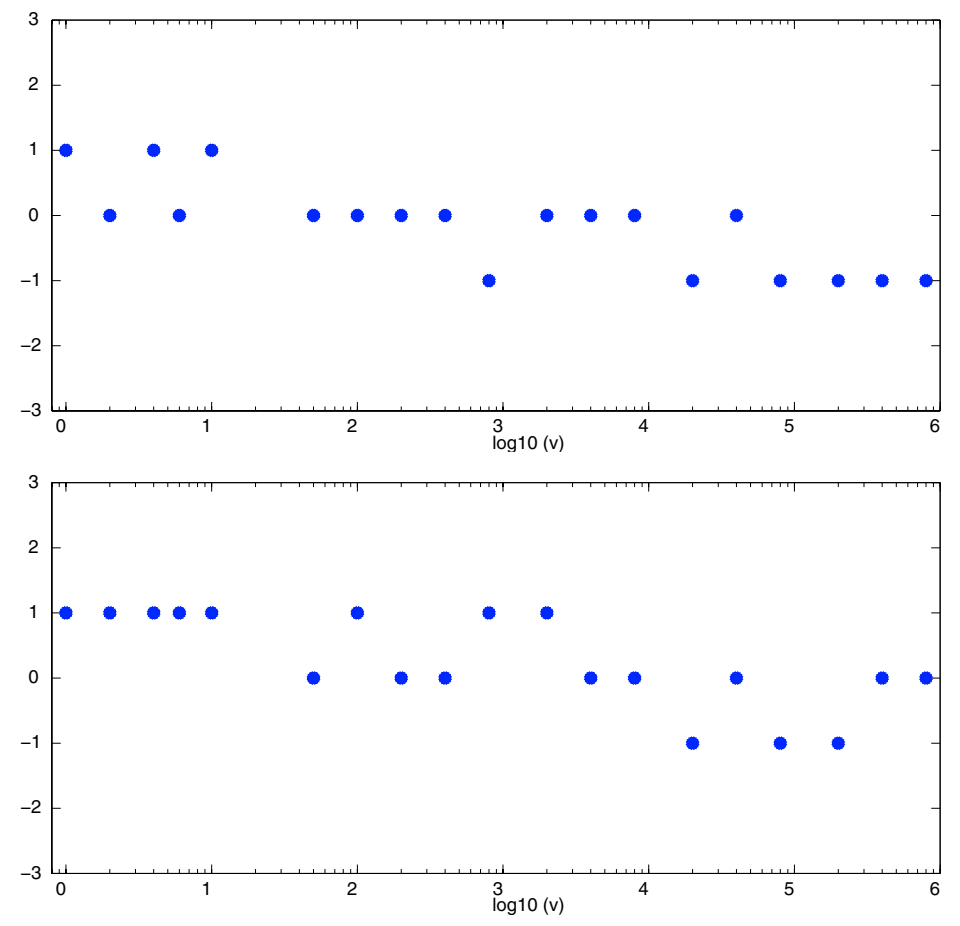

FiguRE 6. Difference between the actual and the estimated truncation integer for $\epsilon=10^{-3}$ (top) and $\epsilon=10^{-7}$ (bottom).

behavior of the error for large values of $v$. The more stringent asymptotic appears to be the one corresponding to $\cos \beta= \pm 1$. In a last paragraph, we prove that the maximum of the error over all the angular apertures $\beta$ is asymptotically reached for the particular value $\cos \beta= \pm 1$.

\subsection{Analysis of the error for a given angular aperture}

In what follows, we will use two different ways to split the real and imaginary parts of the series (10). More precisely, if

$$
\cos \beta=\hat{\mathbf{v}} \cdot \hat{\mathbf{s}},
$$

we define

$$
\left\{\begin{array}{l}
r_{1}(L, \vec{v}, \hat{s})=\left|\sum_{\substack{\ell=+1 \\
\ell \text { even }}}^{\infty}(-1)^{\frac{\ell}{2}}(2 \ell+1) j_{\ell}(v) P_{\ell}(\cos \beta)\right| \\
r_{2}(L, \vec{v}, \hat{s})=\left|\sum_{\substack{\ell=L+1 \\
\ell \text { odd }}}^{\infty}(-1)^{\frac{\ell-1}{2}}(2 \ell+1) j_{\ell}(v) P_{\ell}(\cos \beta)\right|,
\end{array}\right.
$$

and

$$
\left\{\begin{array}{l}
\tilde{r}_{1}(L, \vec{v}, \hat{s})=\sum_{\substack{\ell=L+1+p \\
p \geq 0 \text { even } \\
\infty}}^{\infty}(-1)^{\frac{p}{2}}(2 \ell+1) j_{\ell}(v) P_{\ell}(\cos \beta) \\
\tilde{r}_{2}(L, \vec{v}, \hat{s})=\sum_{\substack{\ell=L+1+p \\
p \geq 0 \text { odd }}}^{\infty}(-1)^{\frac{p-1}{2}}(2 \ell+1) j_{\ell}(v) P_{\ell}(\cos \beta) .
\end{array}\right.
$$


According to whether $L$ is odd or even, $\left(r_{1}, r_{2}\right)$ is $\left(\left|\tilde{r}_{1}\right|,\left|\tilde{r}_{2}\right|\right)$ or $\left(\left|\tilde{r}_{2}\right|,\left|\tilde{r}_{1}\right|\right)$; we distinguish these two splittings only for convenience. In all cases, we have

$$
e_{j a}(L, \vec{v}, \hat{s})=\sqrt{r_{1}(L, \vec{v}, \hat{s})^{2}+r_{2}(L, \vec{v}, \hat{s})^{2}}
$$

and

$$
e_{j a}(L, \vec{v}, \hat{s}) \leq \sqrt{2} \max \left(r_{1}(L, \vec{v}, \hat{s}), r_{2}(L, \vec{v}, \hat{s})\right)
$$

or

$$
e_{j a}(L, \vec{v}, \hat{s}) \leq \sqrt{2} \max \left(\left|\tilde{r}_{1}(L, \vec{v}, \hat{s})\right|,\left|\tilde{r}_{2}(L, \vec{v}, \hat{s})\right|\right) .
$$

3.1.1. The case $|\hat{\mathbf{v}} \cdot \hat{\mathbf{s}}|=0$

We use Bernstein inequality ( 7$)$ with $x=0$ and the fact that $P_{\ell}(0)$ vanishes for odd $\ell\left(r_{2}(L, \vec{v}, \hat{s})\right.$ is therefore zero). We get

$$
e_{j a}(L, v, \hat{s})=r_{1}(L, \vec{v}, \hat{s}) \leq \sqrt{\frac{2}{\pi}} \sum_{\substack{\ell=L+1 \\ \ell \text { even }}}^{\infty} \sqrt{2} \sqrt{2 \ell+1} j_{\ell}(v) .
$$

We assume $v \leq L+\frac{1}{2}$ and simply bound $\sqrt{2 \ell+1}$ by $\frac{2 \ell+1}{\sqrt{2 v}}$ in the summation,

$$
e_{j a}(L, v, \hat{s}) \leq \sqrt{\frac{2}{\pi v}} \sum_{\substack{\ell=L+1 \\ \ell \text { even }}}^{\infty}(2 \ell+1) j_{\ell}(v)=\sqrt{\frac{2}{\pi v}} \begin{cases}\epsilon_{2}(L, v) & L \text { even } \\ \epsilon_{1}(L, v) & L \text { odd }\end{cases}
$$

whence

$$
e_{j a}(L, v, \hat{s}) \leq \sqrt{\frac{2}{\pi v}} \epsilon_{1}(L, v), \quad(\hat{\mathbf{v}} \cdot \hat{\mathbf{s}}=0) .
$$

This estimate is sharper than $e_{j a}(L, v, \hat{s}) \leq e_{j a}^{a b s}(L, v) \leq 2 \epsilon_{1}(L, v)$ when $\frac{1}{2 \pi} \leq v$. It is then easy to follow the steps of Section 2.2 to get an estimate of the truncation integer for which the error is smaller than $\epsilon$. In particular, if $v$ is large, we have

$$
\lim _{\substack{v \rightarrow \infty \\ \cos (\hat{s} \cdot \hat{v})=0}} e_{j a}\left(L^{\epsilon, 0}(v), \vec{v}, \hat{s}\right) \leq \epsilon,
$$

with

$$
L^{\epsilon, 0}(v)+\frac{1}{2}=v+\frac{1}{2}\left(\frac{3}{2}\right)^{\frac{2}{3}} W^{\frac{2}{3}}\left(\frac{1}{3 \pi \epsilon^{2}}\right) v^{\frac{1}{3}} .
$$

We remark that the argument of the Lambert function does not depend on $v$. This means that the empirical formula $L=v+C v^{\frac{1}{3}}$ provides an error asymptotically lower than $\epsilon(C)$ at infinity. This result seems to be optimal according to the results of the numerical experiments ( $c f$. Figs. 2 and 7). Besides, the explicit expressions

$$
P_{\ell}(0)=\left[\begin{array}{ll}
0 & \ell \text { odd } \\
(-1)^{\frac{\ell}{2}} \frac{1 \cdot 3 \cdot 5 \ldots(\ell-1)}{2 \cdot 4 \cdot 6 \ldots \ell} & \ell \text { even }
\end{array}\right.
$$

provide

$$
e_{j a}(L, \vec{v}, \hat{s})=\sum_{\substack{\ell=L+1 \\ \ell \text { even }}}^{\infty}\left(\sqrt{2 \ell+1} \frac{1 \cdot 3 \cdot 5 \ldots(\ell-1)}{2 \cdot 4 \cdot 6 \ldots \ell}\right) \sqrt{2 \ell+1} j_{\ell}(v),
$$

Wallis formula allowing us to replace the term between braces by $2 / \sqrt{\pi}$ with a very good approximation. The main point of this calculation is that all the terms in the series are shown to be positive; therefore, there is no cancellation when adding the terms in the series. It will not be the same in the case $\cos \beta \neq 0$. 


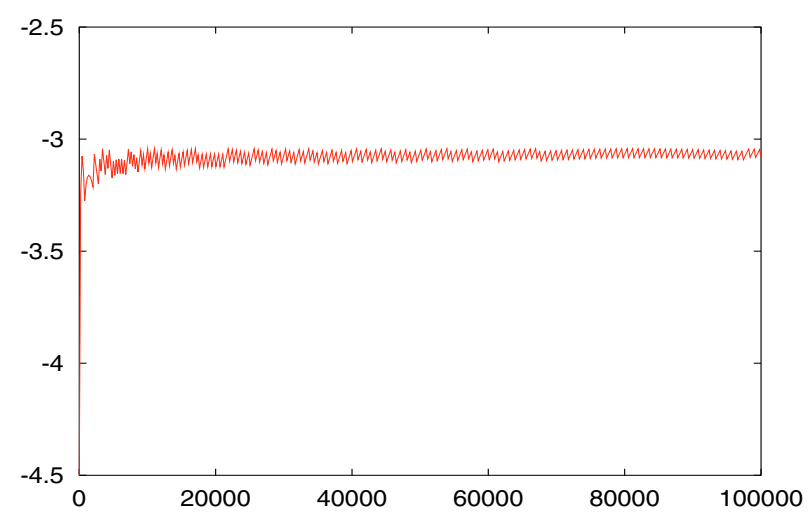

Figure 7. Horizontal axis: $v$. Vertical axis: $\log _{10}\left(e_{j a}(L(v), \vec{v}, \hat{s})\right)$ with $\cos \hat{v} \cdot \hat{s}=0$ and $L(v)=L^{\epsilon=\frac{1}{1000}, 0}(v)$ as given in formula (40).

\subsubsection{The case $0<|\hat{\mathbf{v}} \cdot \hat{\mathbf{s}}|<1$}

To deal with the case $|\hat{\mathbf{v}} \cdot \hat{\mathbf{s}}|<1$, one could use exactly the same steps as previously and get

$$
e_{j a}(L, v, \hat{s}) \leq \frac{2}{\sqrt{\pi v \mid \sin \beta}} \sum_{\substack{\ell=L+1 \\ l \text { even }}}^{\infty}(2 \ell+1) j_{\ell}(v)=\frac{2}{\sqrt{\pi v|\sin \beta|}} \epsilon_{1}(L, v) .
$$

(The $\sqrt{2}$ factor occurring since $r_{2}(L, \vec{v}, \hat{s})$ does not vanish.) We would obtain

$$
\lim _{\substack{v \rightarrow \infty, \cos (\hat{s} \cdot \hat{v})=\beta}} e_{j a}\left(L^{\epsilon, \beta}(v), \vec{v}, \hat{s}\right) \leq \epsilon,
$$

with

$$
L^{\epsilon, \beta}(v)+\frac{1}{2}=v+\frac{1}{2}\left(\frac{3}{2}\right)^{\frac{2}{3}} W^{\frac{2}{3}}\left(\frac{2}{3 \pi|\sin \beta| \epsilon^{2}}\right) v^{\frac{1}{3}} .
$$

However, if $\sin \beta$ is different from zero, a new phenomenon appears. Indeed, in this case, the sequence $P_{\ell}(\cos \beta)$ is, for large $\ell$, an oscillating function in $\ell$ and its period is greater than 2 (period of the term $\left.(-1)^{\ell}\right)$. In the case $\cos \beta=0$, this oscillating property is annihilated by the $(-1)^{\ell}$ factor, but in the other situations, the product of the two sequences is oscillating. This implies that both real and imaginary parts of the terms in the series defining the truncation error are asymptotically alternate (i.e. the generic term of the series decreases in modulus with a periodically change of sign) and the behavior of the series is given by the behavior of its first term. This explains why the numerical results obtained in the case $\cos \beta=0.5$ show a different behavior from the case $\cos \beta=0$.

In what follows, symbol $[x]$ means the integer part of $x$.

Proposition 3.1. If $0<\cos \beta<1$, then

$$
\lim _{\substack{v \rightarrow \infty \\ \cos (\hat{s} \cdot \hat{v})=\cos \beta}} e_{j a}\left(\left[v-\frac{1}{2}\right]+\frac{1}{2}, \vec{v}, \hat{s}\right)=0 .
$$

This proposition means that $L \sim v$ is good enough to ensure that the error goes to zero as $v$ goes to infinity. However, the decrease of the error is very slow: it decreases like $v^{-\frac{1}{3}}$ (see the proof below). This asymptotic behavior can be seen in Figure 8. Moreover, all the constants appearing in the estimate goes to infinity as $|\cos \beta|$ tends to 0 or 1 . 


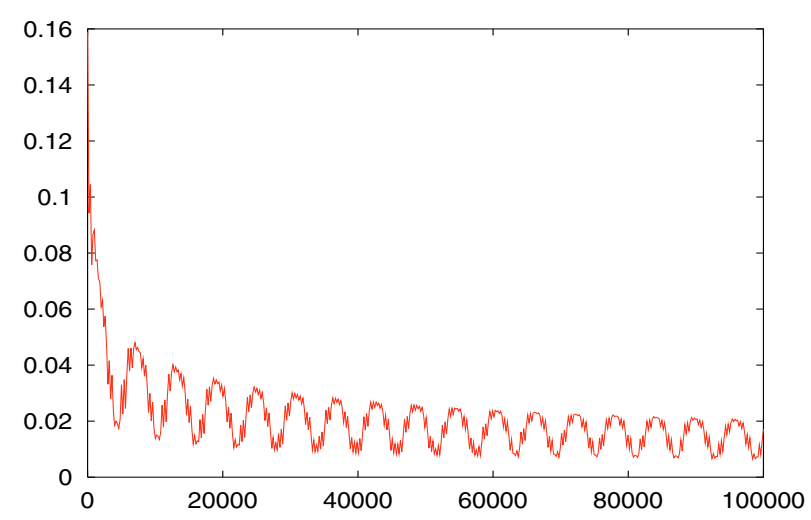

FiguRE 8. Horizontal axis: $v$. Vertical axis: $e_{j a}\left(\left[v+\frac{1}{2}\right], \vec{v}, \hat{s}\right)$ with $\cos \hat{v} \cdot \hat{s}=\cos \beta, \beta=35$ degrees.

Proof. We begin by recalling the Christoffel-Darboux formula ([1], 8.9.1),

$$
(y-x) \sum_{n=0}^{\ell}(2 n+1) P_{n}(x) P_{n}(y)=(\ell+1)\left(P_{\ell+1}(y) P_{\ell}(x)-P_{\ell}(y) P_{\ell+1}(x)\right),
$$

or, setting $x=0, y=\cos \beta$, and assuming $\ell$ to be even

$$
\cos \beta \sum_{\substack{n=0 \\ n \text { even }}}^{\ell}(2 n+1) P_{n}(\cos \beta) P_{n}(0)=(\ell+1) P_{\ell+1}(\cos \beta) P_{\ell}(0) .
$$

Considering $r_{1}(L, \vec{v}, \hat{s})$, we can write

$$
r_{1}(L, \vec{v}, \hat{s})=\left|\sum_{\substack{\ell=L+1 \\ \ell \text { even }}}^{\infty} a_{\ell}(v) b_{\ell}\right|
$$

with

$$
a_{\ell}(v)=\frac{(-1)^{\frac{\ell}{2}}}{P_{\ell}(0)} j_{\ell}(v)(\ell \text { even }), \quad b_{\ell}=(2 \ell+1) P_{\ell}(0) P_{\ell}(\cos \beta) .
$$

Using Bernstein inequality, (44) provides the following estimate

$$
\left|\sum_{\substack{n=0 \\ n \text { even }}}^{\ell} b_{n}\right| \leq \frac{2}{\pi} \frac{\ell+1}{\sqrt{\left(\ell+\frac{1}{2}\right)\left(\ell+\frac{3}{2}\right)}} \frac{1}{|\cos \beta| \sqrt{|\sin \beta|}} \leq \frac{C_{1}}{|\cos \beta| \sqrt{|\sin \beta|}} .
$$

On the other hand, $a_{\ell}(v)$ has a constant sign for even $\ell, \ell+\frac{1}{2} \geq v$, and since

$$
(\ell+2) P_{\ell+2}(0)=-(\ell+1) P_{\ell}(0) \quad \text { and } \quad j_{\ell^{\prime}+1}(v) \leq \frac{v}{\ell^{\prime}+\frac{3}{2}} j_{\ell^{\prime}}(v)
$$

with $\ell^{\prime}=\ell$ or $\ell+1$, we see that

$$
a_{\ell+2}(v) \leq \frac{v^{2}(\ell+2)}{(\ell+1)\left(\ell+\frac{3}{2}\right)\left(\ell+\frac{5}{2}\right)} a_{\ell}(v)
$$

and $a_{\ell=2 k}(v)$ decreases as soon as $\ell-\frac{1}{2} \geq v$. 
We use now the Abel transform. We assume that $L+1$ is even (we replace $L+1$ by $L+2$ otherwise)

$$
\sum_{\substack{\ell=L+1 \\ \ell \text { even }}} a_{\ell}(v) b_{\ell}=\sum_{\substack{\ell=L+1 \\ \ell \text { even }}}\left(a_{\ell}(v)-a_{\ell+2}(v)\right)\left(b_{0}+b_{2}+\cdots+b_{\ell}\right)-a_{L+1}(v)\left(b_{0}+b_{2}+\cdots+b_{L-1}\right) .
$$

Using inequality (45) as well as the decreasing property of the sequence $\left(a_{2 k}\right)$ leads to

$$
\left|\sum_{\substack{\ell=L+1 \\ \ell \text { even }}} a_{\ell}(v) b_{\ell}\right| \leq \frac{C_{1}}{\cos \beta \sqrt{|\sin \beta|}}\left(a_{L+1}+\sum_{\substack{\ell=L+1 \\ \ell \text { even }}}\left(a_{\ell}(v)-a_{\ell+2}(v)\right)\right),
$$

and finally

$$
r_{1}(L, \vec{v}, \hat{s}) \leq \frac{2 C_{1}}{\cos \beta \sqrt{|\sin \beta|}} a_{L+1}(v) .
$$

Since $P_{\ell}(0) \sim \sqrt{\frac{2}{\pi \ell}}$ and $j_{v}(v) \sim C v^{-5 / 6}$, we easily infer that $L=v$ is enough to make $a_{L+1}(v)$ behave like $v^{-\frac{1}{3}}$ as $v$ goes to infinity. Consequently, $v^{\frac{1}{3}} r_{1}(L, \vec{v}, \hat{s})$ is a bounded function.

A similar estimate can be obtained for $r_{2}(L, \vec{v}, \hat{s})$. The identity

$$
(2 \ell+1) P_{\ell}(x)=\frac{\ell+1}{x} P_{\ell+1}(x)+\frac{\ell}{x} P_{\ell-1}(x),
$$

shows that $r_{2}(L, \vec{v}, \hat{s})$ can be split into two sums with Legendre polynomials with even degrees. From this remark, the analysis of $r_{2}(L, \vec{v}, \hat{s})$ can be performed following the same steps as for $r_{1}(L, \vec{v}, \hat{s})$.

\subsubsection{The case $|\hat{\mathbf{v}} \cdot \hat{\mathbf{s}}|=1$}

If $|\hat{\mathbf{v}} \cdot \hat{\mathbf{s}}|=1$, we have $P_{\ell}(1)=1, P_{\ell}(-1)=(-1)^{\ell}$ and the errors for $\beta=0$ and $\pi$ are the same. In these particular cases, the series $(10)$ can be explicited. Let $\varepsilon$ be $1\left(=P_{\ell}(1)\right)$ or $-1\left(=P_{\ell}(-1)\right)$. With the aid of $(17)$, we get

$$
\sum_{\ell=L+1}^{\infty}(2 \ell+1)(\varepsilon i)^{\ell} j_{\ell}(v)=\sqrt{\frac{\pi}{2 v}} \sum_{\ell=L+1}^{\infty}(\varepsilon i)^{\ell}(2 \ell+1) J_{\ell+\frac{1}{2}}(v)=\sqrt{\frac{\pi}{2 v}} \sum_{\ell=L+1}^{\infty}(\varepsilon i)^{\ell} v\left(J_{\ell-\frac{1}{2}}(v)+J_{\ell+\frac{3}{2}}(v)\right),
$$

or

$$
\sum_{\ell=L+1}^{\infty}(2 \ell+1) i^{\ell} j_{\ell}(v)=\sqrt{\frac{\pi v}{2}}(\varepsilon i)^{L+1}\left(J_{L+\frac{1}{2}}(v)+i \varepsilon J_{L+\frac{3}{2}}(v)\right) .
$$

Since $J_{L+\frac{3}{2}}(v) \leq J_{L+\frac{1}{2}}(v)$ for $v<L+\frac{1}{2}$, we obtain

$$
e_{j a}(L, \vec{v}, \hat{s})=\left|\sum_{\ell=L+1}^{\infty}(2 \ell+1)(\varepsilon i)^{\ell} j_{\ell}(v)\right| \leq \sqrt{\pi v} J_{L+\frac{1}{2}}(v),
$$

for $|\hat{\mathbf{v}} \cdot \hat{\mathbf{s}}|=1$ and $0<v<L+\frac{1}{2}$. Watson's bound (22) is then used to get

$$
e_{j a}(L, \vec{v}, \hat{s}) \leq \sqrt{\frac{x}{2}} \frac{\mathrm{e}^{-v \frac{F(x)}{x}}}{\left(1-x^{2}\right)^{\frac{1}{4}}}, \quad x=\frac{v}{L+\frac{1}{2}} .
$$

The term in the right hand side of this inequality is a one-to-one mapping in $x$ from $] 0,1[$ onto $] 0,+\infty[$. We obtain once again a practical criterion for the truncation by dichotomy. For large $v, F(x)$ is bounded from 


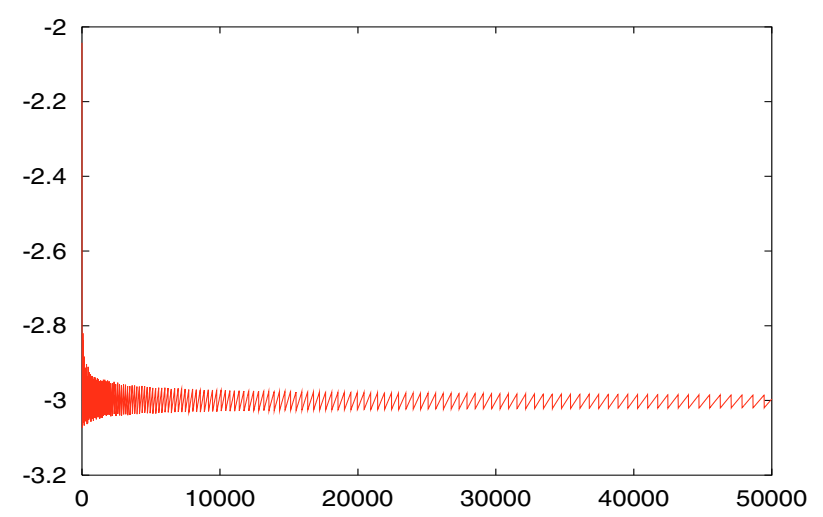

FiguRe 9. Horizontal axis: $v$. Vertical axis: $\log _{10}\left(e_{j a}(L(v), \vec{v}, \hat{s})\right)$ with $|\cos (\vec{v} \cdot \hat{s})|=1$ and $L(v)=L^{\epsilon=\frac{1}{1000}, \pi}(v)$ as given in formula (52).

below by $\frac{1}{3}\left(1-x^{2}\right)^{\frac{3}{2}}=\frac{x}{3} \Theta(x)$, and replacing $\sqrt{x}$ by the larger quantity $x^{\frac{1}{6}}$ in (48) yields

$$
e_{j a}(L, \vec{v}, \hat{s}) \leq \sqrt{\pi v} J_{L+\frac{1}{2}}(v) \leq \frac{\mathrm{e}^{-\frac{v}{3} \Theta(x)}}{\sqrt{2} \Theta(x)^{\frac{1}{6}}}, \quad(|\hat{\mathbf{v}} \cdot \hat{\mathbf{s}}|=1) .
$$

Proceeding in the same way as in Section 2.2, we obtain

$$
\frac{\mathrm{e}^{-\frac{v}{3} \Theta(x)}}{\sqrt{2} \Theta(x)^{\frac{1}{6}}}=\epsilon \quad \Leftrightarrow \quad \Theta(x)=\frac{1}{2 v} W\left(\frac{2 v}{(\sqrt{2} \epsilon)^{6}}\right)
$$

As $v$ approaches infinity, $x$ approaches 1 and we get the asymptotic

$$
\lim _{\substack{v \rightarrow \infty \\(\hat{s}, \hat{v})= \pm 1}} e_{j a}\left(L^{\epsilon, \pi}(v), \vec{v}, \hat{s}\right) \leq \epsilon
$$

with

$$
L^{\epsilon, \pi}(v)+\frac{1}{2}=v+\left(\frac{1}{2}\right)^{\frac{5}{3}} W^{\frac{2}{3}}\left(\frac{v}{4 \epsilon^{6}}\right) v^{\frac{1}{3}}
$$

This result corresponds to what we observe in Figure 2, since using the empirical formula $L=v+C v^{\frac{1}{3}}$ amounts to taking the argument of the Lambert function constant, and so to having an error varying like $v^{\frac{1}{6}}$ when $v$ is large. The accuracy of our estimate is confirmed by the numerical computations presented in Figure 9.

\subsection{A uniform estimate}

We show in this paragraph that it is possible to improve the estimate of the truncation found in Section 2. In particular, the truncation (52), which provides asymptotically an error $\epsilon$ for two parallel vectors $\hat{s}$ and $\vec{v}$, is found to give an asymptotic error lower than $\epsilon$ uniformly for all $\hat{s}$. We note that this result cannot be inferred directly from (41)-(42) since $L^{\epsilon, \beta}(v)$ goes to infinity as $\beta$ tends to zero. 
We start with $\tilde{r}_{1}(L, \vec{v}, \hat{\mathbf{s}})$

$$
\begin{aligned}
\tilde{r}_{1}(L, \vec{v}, \hat{\mathbf{s}})= & \sqrt{\frac{\pi}{2 v}} \sum_{\substack{\ell=L+1+p \\
p=0(4)}}^{\infty}(2 \ell+1) J_{\ell+\frac{1}{2}}(v) P_{\ell}(\hat{\mathbf{v}} \cdot \hat{\mathbf{s}})-(2 \ell+5) J_{\ell+\frac{5}{2}}(v) P_{\ell+2}(\hat{\mathbf{v}} \cdot \hat{\mathbf{s}}) \\
= & \sqrt{\frac{\pi}{2 v}} \sum_{\substack{\ell=L+1+p \\
p=0(4)}}^{\infty}\left[(2 \ell+1) J_{\ell+\frac{1}{2}}(v)-(2 \ell+5) J_{\ell+\frac{5}{2}}(v)\right] P_{\ell}(\hat{\mathbf{v}} \cdot \hat{\mathbf{s}}) \\
& +\sqrt{\frac{\pi}{2 v}} \sum_{\substack{\ell=L+1+p \\
p=0(4)}}^{\infty}(2 \ell+5) J_{\ell+\frac{5}{2}}(v)\left[P_{\ell}(\hat{\mathbf{v}} \cdot \hat{\mathbf{s}})-P_{\ell+2}(\hat{\mathbf{v}} \cdot \hat{\mathbf{s}})\right] .
\end{aligned}
$$

In the first sum, we bound the Legendre polynomials by 1 and use (17)

$$
\left|\sum_{\substack{\ell=L+1+p \\ p \equiv 0(4)}}^{\infty}\left[(2 \ell+1) J_{\ell+\frac{1}{2}}(v)-(2 \ell+5) J_{\ell+\frac{5}{2}}(v)\right] P_{\ell}(\hat{\mathbf{v}} \cdot \hat{\mathbf{s}})\right| \leq \sum_{\substack{\ell=L+1+p \\ p=0(4)}}^{\infty}\left|v J_{\ell-\frac{1}{2}}(v)-v J_{\ell+\frac{7}{2}}(v)\right| .
$$

Due to the decrease of $J_{\nu}(v)$ in $\nu$ for $v<\nu$, we can remove the absolute values; this gives

$$
v \sum_{\substack{\ell L+1+p \\ p \equiv 0(4)}}^{\infty}\left(J_{\ell-\frac{1}{2}}(v)-J_{\ell+\frac{7}{2}}(v)\right)=v J_{L+\frac{1}{2}}(v)
$$

In the second sum, we use the inequality known as the Stieltjes inequality (cf. [13], p. 1047)

$$
\left.\left.\exists C_{0} \in\right] 0, \frac{2}{\sqrt{\pi}}\right]: \quad \forall \ell>0, \quad \forall x \in[-1,1], \quad\left|P_{\ell}(x)-P_{\ell+2}(x)\right| \leq \frac{2 C_{0}}{\sqrt{\ell}} .
$$

Then, we obtain

$$
\left|\tilde{r}_{1}(L, \vec{v}, \hat{\mathbf{s}})\right| \leq \sqrt{\frac{\pi v}{2}} J_{L+\frac{1}{2}}(v)+2 C_{0} \sqrt{\frac{\pi}{2 v}} \sum_{\substack{\ell=L+1+p \\ p \equiv(4)}}^{\infty} \frac{2 \ell+5}{\sqrt{\ell}} J_{\ell+\frac{5}{2}}(v) .
$$

By using the decrease of the sequence $\left(\nu J_{\nu}(v)\right)_{\nu>v}$ (see (19)), the series appearing in the above inequality can be estimated like

$$
\begin{gathered}
\sum_{\substack{\ell=L+1+p \\
p=0(4)}}^{\infty} \frac{2 \ell+5}{\sqrt{\ell}} J_{\ell+\frac{5}{2}}(v) \leq \frac{1}{\sqrt{v}} \sum_{\substack{\ell=L+1+p \\
p=0(4)}}^{\infty} 2\left(\ell+\frac{5}{2}\right) J_{\ell+\frac{5}{2}}(v) \\
\leq \frac{1}{\sqrt{v}} \sum_{\substack{\ell=L+1+p \\
p \equiv(4)}}^{\infty}\left[\left(\ell+\frac{5}{2}\right) J_{\ell+\frac{5}{2}}(v)+\left(\ell+\frac{3}{2}\right) J_{\ell+\frac{3}{2}}(v)\right] \leq \frac{1}{\sqrt{v}} \sum_{\substack{\ell=L+2+p \\
p \text { even }}}^{\infty}\left(\ell+\frac{1}{2}\right) J_{\ell+\frac{1}{2}}(v) .
\end{gathered}
$$

This gives, according to (16),

$$
2 C_{0} \sqrt{\frac{\pi}{2 v}} \sum_{\substack{\ell=L+1+p \\ p=0(4)}}^{\infty} \frac{2 \ell+5}{\sqrt{\ell}} J_{\ell+\frac{5}{2}}(v) \leq \frac{C_{0}}{\sqrt{v}} \epsilon_{1}(L+1, v) \leq \frac{C_{0}}{\sqrt{v}} \epsilon_{1}(L, v) .
$$

From this estimate, we are led to

$$
\left|\tilde{r}_{1}(L, \vec{v}, \hat{\mathbf{s}})\right| \leq \sqrt{\frac{\pi v}{2}} J_{L+\frac{1}{2}}(v)+\frac{C_{0}}{\sqrt{v}} \epsilon_{1}(L, v) .
$$


Following the same procedure, one can obtain a similar bound for $\left|\tilde{r}_{2}(L, \vec{v}, \hat{\mathbf{s}})\right|$.

Finally, we get

$$
e_{j a}(L, \vec{v}, \hat{s}) \leq \sqrt{\pi v} J_{L+\frac{1}{2}}(v)+C_{0} \sqrt{\frac{2}{v}} e_{j a}^{a b s}(L, v)
$$

This estimate improves the bound $e_{j a}(L, \vec{v}, \hat{s}) \leq e_{j a}^{a b s}(L, v)$ due to the factor $\frac{1}{\sqrt{v}}$ in front of $e_{j a}^{a b s}(L, v)$ and also since $\sqrt{v} J_{L+\frac{1}{2}}(v)$ decreases faster than $e_{j a}^{a b s}(L, v)$. Note that the first term corresponds to the error for two parallel vectors.

We set $x=\frac{v}{L+\frac{1}{2}}, \Theta(x)=x^{-1}\left(1-x^{2}\right)^{\frac{3}{2}}$, and use (30) and (49). Thus, we have

$$
e_{j a}(L, \vec{v}, \hat{s}) \leq \frac{\mathrm{e}^{-\frac{v}{3} \Theta(x)}}{\sqrt{2} \Theta(x)^{\frac{1}{6}}}\left(1+\frac{2 C_{0}}{\sqrt{v} \Theta(x)^{\frac{1}{3}}}\right) .
$$

If $\Theta(x)$ is chosen as in (50), we obtain

$$
e_{j a}(L, \vec{v}, \hat{s}) \leq \epsilon\left(1+\frac{2^{\frac{1}{3}} 2 C_{0}}{v^{\frac{1}{6}} W^{\frac{1}{3}}\left(\frac{v}{4 \epsilon^{6}}\right)}\right),
$$

and the term between braces tends to 1 when $v$ goes to infinity. Whence we proved the proposition:

Proposition 3.2. Let $\vec{v}, \vec{s}$ be 2 vectors in $\mathbf{R}^{3}, v=|\vec{v}|,|\vec{s}|=1$ and $\epsilon$ be a positive number, let us set

$$
L_{0}^{\epsilon, \infty}(v)+\frac{1}{2}=v+\left(\frac{1}{2}\right)^{\frac{5}{3}} W^{\frac{2}{3}}\left(\frac{v}{4 \epsilon^{6}}\right) v^{\frac{1}{3}}
$$

and let us define

then we have

$$
\left\|e_{j a}(L, \vec{v}, .)\right\|_{\infty}=\sup _{\hat{s}}\left|\sum_{\ell=L+1}^{\infty}(2 \ell+1) i^{\ell} j_{\ell}(v) P_{\ell}(\hat{\mathbf{v}} \cdot \hat{\mathbf{s}})\right|
$$

$$
\lim _{v \rightarrow \infty}\left\|e_{j a}\left(L_{0}^{\epsilon, \infty}(v), \vec{v}, .\right)\right\|_{\infty} \leq \epsilon .
$$

This result is illustrated in Figure 10 where, for a given $v=1000$, we plot in polar coordinates the error $e_{j a}\left(L_{0}^{\epsilon, \infty}(v), \vec{v},.\right)$ versus the angle of aperture $\beta$. The error reaches its maximum value for $\beta=0$ and $\pi$ and a local maxima can be seen for $\beta= \pm \frac{\pi}{2}$. This numerical result agrees with our analysis.

Remark 3.3. The increase in $v^{1 / 6}$ of the error when using laws in the form $L=v+C v^{1 / 3}$ is a 3 -D phenomenon: in 2-D, assuming $L>v$

$$
\left|\mathrm{e}^{i v \cos \theta}-\sum_{\ell=-L}^{L} i^{\ell} J_{\ell}(v) \mathrm{e}^{i \ell \theta}\right|=2\left|\sum_{\ell>L} i^{\ell} J_{\ell}(v) \cos \ell \theta\right|=2\left|\sum_{\substack{\ell>L \\ \ell=2 p}}(-1)^{p} J_{\ell}(v) \cos \ell \theta+i \sum_{\substack{\ell>L \\ \ell=2 p+1}}(-1)^{p} J_{\ell}(v) \cos \ell \theta\right|,
$$

then using the decrease of the sequence $\left(J_{\ell}(v)\right)_{\ell>v}$,

$$
\left|\mathrm{e}^{i v \cos \theta}-\sum_{\ell=-L}^{L} i^{\ell} J_{\ell}(v) \mathrm{e}^{i \ell \theta}\right| \leq 2 \sqrt{2} \sum_{\substack{\ell=L+1+2 p \\ p \geq 0}} J_{\ell}(v) \leq \sqrt{2} \sum_{\substack{\ell=L+1+2 p \\ p \geq 0}} \frac{2 \ell}{v} J_{\ell}(v)
$$

the last term is $\frac{2}{\sqrt{\pi v}} \epsilon_{1}\left(L-\frac{1}{2}, v\right)$, hence, following the steps of Section $2.2, L \sim v+\frac{1}{2}\left(\frac{3}{2}\right)^{\frac{2}{3}} W^{\frac{2}{3}}\left(\frac{2}{3 \pi \epsilon^{2}}\right) v^{\frac{1}{3}}$ gives an error asymptotically less than $\epsilon$ when $v$ is large (this agrees with the results in [18]). 


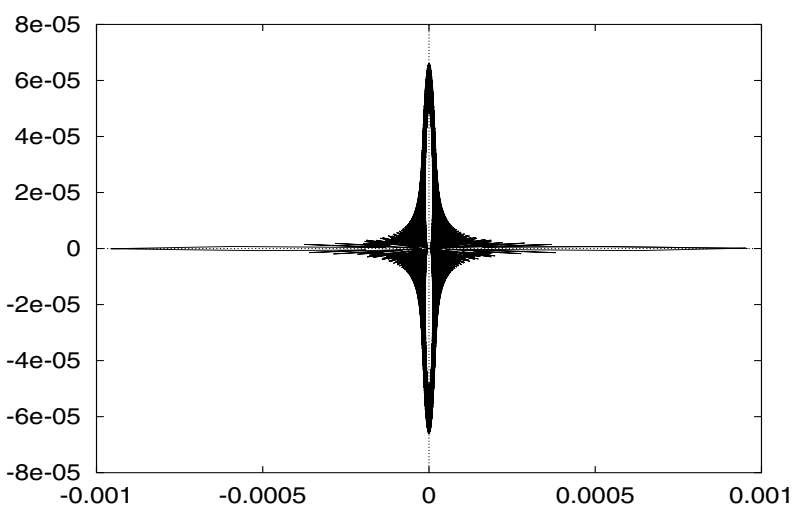

Figure 10. Curve $\beta \rightarrow e^{i \beta} \log _{10}\left(e_{j a}(L, \vec{v}, \hat{s}(\beta))\right)$ with $\vec{v}=v \hat{v}, \hat{v} \cdot \hat{s}(\beta)=\cos \beta . v=1000$ and $L=1038=L_{0}^{\epsilon=\frac{1}{1000}, \infty}$, see (55).

\subsection{Analysis of the error in $L^{2}$}

We complete this paper by a short study of the relative error in $L^{2}\left(S^{2}\right)$. We define

$$
e_{2}^{j a}(L, v)=\frac{1}{4 \pi} \int_{S^{2}}\left|e_{j a}(L, \vec{v}, \hat{s})\right|^{2} \mathrm{~d} \sigma(\hat{s}) .
$$

Since the Legendre polynomials satisfy

$$
\int_{S^{2}} P_{n}(\hat{\mathbf{v}} \cdot \hat{\mathbf{s}}) P_{m}(\hat{\mathbf{v}} \cdot \hat{\mathbf{s}}) \mathrm{d} \sigma_{s}=\frac{4 \pi}{2 n+1} \delta_{n}^{m}
$$

it is possible to transform this expression into

$$
e_{2}^{j a}(L, v)=\sum_{n=L+1}^{+\infty}(2 n+1) j_{n}^{2}(v)
$$

The following formula can be found in ([7], p. 61)

$$
(2 n+1) j_{n}^{2}(v)=\frac{1}{v} \int_{0}^{v} \tau^{2}\left[j_{n-1}^{2}(\tau)-j_{n+1}^{2}(\tau)\right] \mathrm{d} \tau .
$$

Substituting in (57) gives after simplication

$$
e_{2}^{j a}(L, v)=\frac{1}{v}\left\{\int_{0}^{v} \tau^{2} j_{L}^{2}(\tau) \mathrm{d} \tau+\int_{0}^{v} \tau^{2} j_{L+1}^{2}(\tau) \mathrm{d} \tau,\right\}
$$

or in an equivalent manner

$$
e_{2}^{j a}(L, v)=\frac{\pi}{2 v}\left\{\int_{0}^{v} \tau J_{L+\frac{1}{2}}^{2}(\tau) \mathrm{d} \tau+\int_{0}^{v} \tau J_{L+\frac{3}{2}}^{2}(\tau) \mathrm{d} \tau\right\} .
$$

The integrals can be rewritten in an explicit form, since ([21], p. 149)

$$
\int_{0}^{v} \tau J_{\nu}^{2}(\tau) \mathrm{d} \tau=\frac{v^{2}}{2}\left(J_{\nu}^{2}(v)-J_{\nu+1}(v) J_{\nu-1}(v)\right)
$$


whence

$$
e_{2}^{j a}(L, v)=\frac{\pi v}{4}\left(J_{L+\frac{1}{2}}^{2}(v)-J_{L+\frac{3}{2}}(v) J_{L-\frac{1}{2}}(v)\right)+\frac{\pi v}{4}\left(J_{L+\frac{3}{2}}^{2}(v)-J_{L+\frac{5}{2}}(v) J_{L+\frac{1}{2}}(v)\right) .
$$

Using these formulas, we prove the following proposition:

Proposition 3.4. If

then we have

$$
L(v)=\left[v+\frac{1}{2}\right]
$$

$$
\lim _{v \rightarrow \infty} \int_{S^{2}}\left|\sum_{\ell=L(v)+1}^{\infty}(2 \ell+1) i^{\ell} j_{\ell}(v) P_{\ell}(\hat{\mathbf{v}} \cdot \hat{\mathbf{s}})\right|^{2} \mathrm{~d} \sigma(\hat{s})=0 .
$$

In other words, the truncation error measured in the $L^{2}\left(S^{2}\right)$ norm goes to 0 when the truncation is done at $L \sim v$, which is completely different from the results for the $L^{\infty}\left(S^{2}\right)$ norm.

Proof. we resume (58) and take $L=\left[v+\frac{1}{2}\right], \nu=L+\frac{1}{2}=\left[v+\frac{1}{2}\right]+\frac{1}{2}$. Let $\gamma$ be some real number in the interval ]$\frac{1}{3}, \frac{2}{3}[$. We can write

$$
\begin{aligned}
& \frac{1}{v} \int_{0}^{v} \tau J_{\nu}^{2}(\tau) \mathrm{d} \tau=\frac{1}{v} \int_{0}^{v-v^{\gamma}} \tau J_{\nu}^{2}(\tau) \mathrm{d} \tau+\frac{1}{v} \int_{v-v^{\gamma}}^{v} \tau J_{\nu}^{2}(\tau) \mathrm{d} \tau \\
& =\quad I_{1}(v)+I_{2}(v) .
\end{aligned}
$$

We consider the two terms $I_{1}(v)$ and $I_{2}(v)$ separately.

Term $\mathbf{I}_{\mathbf{1}} . J_{\nu}(\tau)$ is an increasing function in $\tau$ on $[0, \nu]$ :

$$
\text { if } 0 \leq \tau \leq v-v^{\gamma}, \quad \tau J_{\nu}^{2}(\tau)<v J_{\nu}^{2}\left(v-v^{\gamma}\right),
$$

and since $\nu=\left[v+\frac{1}{2}\right]+\frac{1}{2} \geq v$ and $v^{\gamma} \leq v$ for large $v$

$$
I_{1}(v) \leq\left(v-v^{\gamma}\right) J_{\nu}^{2}\left(v-v^{\gamma}\right) \leq v J_{\nu}^{2}\left(\frac{\nu}{v}\left(v-v^{\gamma}\right)\right) .
$$

If $s=\frac{v-v^{\gamma}}{v}=1-v^{\gamma-1}$, Watson's bound (22) gives,

$$
J_{\nu}(\nu s) \leq \frac{\mathrm{e}^{-\frac{\nu}{3}\left(1-s^{2}\right)^{\frac{3}{2}}}}{\left(1-s^{2}\right)^{\frac{1}{4}} \sqrt{2 \pi \nu}} \leq \frac{\mathrm{e}^{-\frac{v}{3}\left(1-s^{2}\right)^{\frac{3}{2}}}}{\left(1-s^{2}\right)^{\frac{1}{4}} \sqrt{2 \pi v}} .
$$

Since $v\left(1-s^{2}\right)^{\frac{3}{2}} \sim 2^{\frac{3}{2}} v^{\frac{1}{2}(3 \gamma-1)}$ and $\left(1-s^{2}\right)^{\frac{1}{4}} \sim 2^{\frac{1}{4}} v^{\frac{\gamma-1}{4}}$, we have for $v$ large

$$
v J_{\nu}^{2}\left(\frac{\nu}{v}\left(v-v^{\gamma}\right)\right) \leq C v^{\frac{1-\gamma}{2}} \mathrm{e}^{-\frac{2}{3} 2^{\frac{3}{2}} v^{\frac{3 \gamma-1}{2}}},
$$

which goes to 0 as $v$ goes to infinity if $\gamma$ is larger than $\frac{1}{3}$. Finally

$$
\lim _{v \rightarrow+\infty} I_{1}(v)=0
$$

Term $\mathbf{I}_{\mathbf{2}}$. We use once again the increase of the function $J_{\nu}(v)$ in $v$

$$
\text { if } v-v^{\gamma} \leq \tau \leq v \leq \nu, \quad \tau J_{\nu}^{2}(\tau)<v J_{\nu}^{2}(v),
$$


and after integration:

$$
I_{2}(v) \leq v^{\gamma} J_{\nu}^{2}(v) \leq \nu^{\gamma} J_{\nu}^{2}(\nu)
$$

As we have the equivalence $J_{\nu}(\nu) \sim \frac{2^{\frac{1}{3}}}{3^{\frac{2}{3}} \Gamma\left(\frac{2}{3}\right)} \nu^{-\frac{1}{3}}$ for $\nu$ large, we readily obtain

$$
I_{2}(v) \leq C v^{\gamma-\frac{2}{3}} \Rightarrow \lim _{v \rightarrow+\infty} I_{2}(v)=0,
$$

if $\gamma$ is smaller than $\frac{2}{3}$. The proof is complete.

\section{Conclusion}

We proved that:

- bounding $\sum_{\ell=L+1}^{\infty}(2 \ell+1)\left|j_{\ell}(v)\right|$ by $\epsilon$ for $v$ large can be accurately obtained when

$$
L \simeq v+\frac{1}{2}\left(\frac{3}{2}\right)^{2 / 3} W^{2 / 3}\left(\frac{2 v}{3 \epsilon^{2}}\right) v^{1 / 3}-\frac{1}{2}+\text { terms vanishing when } v \text { is large; }
$$

- bounding $\left|\sum_{\ell=L+1}^{\infty}(2 \ell+1) i^{\ell} j_{\ell}(v) P_{\ell}(\hat{\mathbf{v}} \cdot \hat{\mathbf{s}})\right|$ by $\epsilon$ for $v$ large can be obtained when

$$
L \simeq v+\left(\frac{1}{2}\right)^{5 / 3} W^{2 / 3}\left(\frac{v}{4 \epsilon^{6}}\right) v^{1 / 3}-\frac{1}{2}+\text { terms vanishing when } v \text { is large; }
$$

- The angular configurations $(\hat{\mathbf{v}}, \hat{\mathbf{s}})$ satisfying to $|\hat{\mathbf{v}} \cdot \hat{\mathbf{s}}|=1$ are the most penalizing to bound the remainder of the Jacobi-Anger series for large $v$.

For the first time, these results show the true behaviour of the error induced by some of the FMM formulas, (especially when the size of the cluster of points that are gathered in the FMM is very large.) Our result contradicts the empirical laws used practically: this is certainly due to the fact that our asymptotic differs from the usual one only in the case of extremely large clusters (large $v$ in our notations).

This first paper does not answer to all the questions about truncation errors in FMM. However all the mathematical tools and results developed here are now available to establish similar formulas for the Gegenbauer series. For instance, in the second part of our work, we will prove that the same kind of bounds can be accurately obtained, for the relative error on the Gegenbauer expansion with finite $u$ when

$$
L \simeq v+C W^{2 / 3}\left(K(\alpha) \frac{v^{\gamma}}{\epsilon^{\delta}}\right) v^{1 / 3}-\frac{1}{2}+\text { terms vanishing when } v \text { is large with } \alpha=\frac{u}{v} .
$$

The fact that the constant $K$ depends on $\alpha=\frac{u}{v}$ is the main difference with the Jacobi-Anger series.

\section{REFERENCES}

[1] M. Abramowitz and I. Stegun, Handbook of Mathematical Functions. Dover, New York (1964).

[2] S. Amini and A. Profit, Analysis of the truncation errors in the fast multipole method for scattering problems. J. Comput. Appl. Math. 115 (2000) 23-33.

[3] Q. Carayol, Développement et analyse d'une méthode multipôle multiniveau pour l'électromagnétisme. Ph.D. Thesis, Université Paris VI Pierre et Marie Curie, Paris (2002).

[4] O. Cessenat and B. Després, Application of an ultra weak variational formulation of elliptic pdes to the 2D Helmholtz problem. SIAM J. Numer. Anal. 35 (1998) 255-299.

[5] W.C. Chew, J.M. Jin, E. Michielssen and J.M. Song, Fast and Efficient Algorithms in Computational Electromagnetics. Artech House (2001). 
[6] R. Coifman, V. Rokhlin and S. Greengard, The fast multipole method for the wave equation: A pedestrian prescription. IEEE Antennas and Propagation Magazine 35 (1993) 7-12.

[7] D. Colton and R. Kress, Inverse Acoustic and Electromagnetic Scattering Theory. Springer-Verlag 93 (1992).

[8] R. Corless, G.H. Gonnet, D.E.G. Hare, D.J. Jeffrey and D.E. Knuth, On the Lambert $W$ function. Adv. Comput. Math. 5 (1996) 329-359.

[9] E. Darve, The fast multipole method. I. Error analysis and asymptotic complexity. SIAM J. Numer. Anal. 38 (2000) 98-128 (electronic).

[10] E. Darve, The fast multipole method: Numerical implementation. J. Comput. Phys. 160 (2000) 196-240.

[11] E. Darve and P. Havé, Efficient fast multipole method for low frequency scattering. J. Comput. Phys. (to appear).

[12] M.A. Epton and B. Dembart, Multipole translation theory for the three-dimensional Laplace and Helmholtz equations. SIAM J. Sci. Comput. 16 (1995) 865-897.

[13] I.S. Gradshteyn and I.M. Ryzhik, Table of integrals, series, and products, 5th edn., Academic Press (1994).

[14] S. Koc, J. Song and W.C. Chew, Error analysis for the numerical evaluation of the diagonal forms of the scalar spherical addition theorem. SIAM J. Numer. Anal. 36 (1999) 906-921 (electronic).

[15] L. Lorch, Alternative proof of a sharpened form of Bernstein's inequality for Legendre polynomials. Applicable Anal. 14 (1982/83) 237-240.

[16] L. Lorch, Corrigendum: "Alternative proof of a sharpened form of Bernstein's inequality for Legendre polynomials" [Appl. Anal. 14 (1982/83) 237-240; MR 84k:26017]. Appl. Anal. 50 (1993) 47.

[17] J.C. Nédélec, Acoustic and Electromagnetic Equation. Integral Representation for Harmonic Problems. Springer-Verlag 144 (2001).

[18] S. Ohnuki and W.C. Chew, Numerical accuracy of multipole expansion for 2-d mlfma. IEEE Trans. Antennas Propagat. 51 (2003) 1883-1890.

[19] J. Rahola, Diagonal forms of the translation operators in the fast multipole algorithm for scattering problems. BIT 36 (1996) 333-358.

[20] G.N. Watson, Bessel functions and Kapteyn series. Proc. London Math. Soc. (1916) 150-174.

[21] G.N. Watson, A treatise on the theory of Bessel functions. Cambridge University Press (1966).

To access this journal online:

www.edpsciences.org 